\title{
CONFORMALLY FLAT HOMOGENEOUS PSEUDO-RIEMANNIAN FOUR-MANIFOLDS
}

\author{
Giovanni Calvaruso AND Amirhesam Zaeim
}

(Received September 25, 2012, revised February 26, 2013)

\begin{abstract}
We obtain a complete classification of four-dimensional conformally flat homogeneous pseudo-Riemannian manifolds.
\end{abstract}

1. Introduction. Conformally flat manifolds are a classical field of investigation in pseudo-Riemannian geometry. In this framework, it is a natural problem to classify conformally flat homogeneous pseudo-Riemannian manifolds. A conformally flat (locally) homogeneous Riemannian manifold is (locally) symmetric [17]. Hence, it admits as univeral covering either a space form $\boldsymbol{R}^{n}, \boldsymbol{S}^{n}(k), \boldsymbol{H}^{n}(-k)$, or one of Riemannian products $\boldsymbol{R} \times \boldsymbol{S}^{n-1}(k), \boldsymbol{R} \times$ $\boldsymbol{H}^{n-1}(-k), \boldsymbol{S}^{p}(k) \times \boldsymbol{H}^{n-p}(-k)[13]$.

In pseudo-Riemannian settings, the problem of classifying conformally flat homogeneous manifolds is more complicated and interesting. Three-dimensional examples were classified independently in [8] and [3], showing the existence of non-symmetric examples. Using the general results introduced in [8], the same authors contributed in [9] to solve the classification problem for Lorentzian manifolds of any dimension, under some assumptions on the structure of the eigenvalues of the Ricci operator of such a manifold. Up to our knowledge, no classification results have been obtained yet for metrics of different signatures, except for the cases with a diagonalizable Ricci operator [8].

In the present paper we shall provide a complete classification of four-dimensional conformally flat homogeneous pseudo-Riemannian manifolds. A fundamental step for this classification will be to understand which forms (Segre types) of the Ricci operator, and under which restrictions, may exist for conformally flat pseudo-Riemannian manifolds. As we shall see, nondegenerate forms of the Ricci operator can only occur when a conformally flat homogeneous pseudo-Riemannian four-manifold is (locally) isometric to some Lie group, while the possible degenerate forms are also realized by some homogeneous spaces with nontrivial isotropy, for which we can use Komrakov's classification [10] to deduce all possible examples.

Because of the results obtained in [9], we shall focus mainly on the case of a pseudoRiemannian metric of neutral signature. However, we shall also explain how our results apply to the Lorentzian case.

The paper is organized in the following way. In Section 2, we report some basic information on four-dimensional conformally flat pseudo-Riemannian manifolds and describe

2000 Mathematics Subject Classification. Primary 53C50; Secondary 53C15, 53C25.

Key words and phrases. Conformally flat manifolds, homogeneous pseudo-Riemannian manifolds, Ricci operator, Segre types. 
the possible forms of the Ricci operator at an arbitrary point. In Section 3, we show that a fundamental distinction arises in terms of isotropy between the cases with nondegenerate and degenerate Ricci operator (Theorem 3.1). Two different approaches will be then used in Sections 3 (for the examples with nondegenerate Ricci operator) and 4 (for the examples with degenerate Ricci operator) when the isotropy is trivial, and in Section 5 to classify homogeneous examples with degenerate Ricci operator and nontrivial isotropy.

Acknowledgments. The authors wish to express their sincere gratitude to the Referee for the valuable and useful comments. The first author was partially supported by funds of the University of Salento and MIUR (PRIN 2012). This work was prepared during the stay of the second author at the University of Salento. The second author wishes to thank the Department of Mathematics of the University of Salento for the hospitality.

2. Conformally flat homogeneous four-manifolds. Let $M_{q}^{n}$ be a pseudo-Riemannian manifold of index $q$. The Weyl conformal curvature tensor fields $C$ of type $(1,3)$ and $c$ of type $(1,2)$ of $M$ are defined by

$$
C(X, Y) Z=R(X, Y) Z-\frac{1}{n-2}(Q X \wedge Y+X \wedge Q Y) Z+\frac{S}{(n-1)(n-2)}(X \wedge Y) Z
$$

and

$$
c(X, Y)=\left(\nabla_{X} Q\right) Y-\left(\nabla_{Y} Q\right) X-\frac{1}{2(n-1)}(X(S) Y-Y(S) X),
$$

respectively. In these equations, $R, Q$ and $S$ respectively denote the curvature tensor, the Ricci operator and the scalar curvature, and $(X \wedge Y)(Z)=\langle Y, Z\rangle X-\langle X, Z\rangle Y$. When $C=0$, the equation (2.1) yields that the Ricci curvature completely determines the curvature of $(M, g)$. It is well known that

- if $n \geq 4$, then $M_{q}^{n}$ is conformally flat if and only if $C=0$, in this case, $c=0$,

- if $n=3$, then $C=0$, and $M$ is conformally flat if and only if $c=0$.

Let now $(M, g)$ be a locally homogeneous pseudo-Riemannian manifold. Then, for each pair of points $p, p^{\prime} \in M$, there exists a local isometry $f$ between neighbourhoods of $p$ and $p^{\prime}$, such that $f(p)=p^{\prime}$. In particular, for any choice of an index $k, f^{*}: T_{p^{\prime}} M \rightarrow T_{p} M$ satisfies $f^{*}\left(\nabla^{i} R_{p^{\prime}}\right)=\nabla^{i} R_{p}$ for all $i=0, \ldots, k$. Consequently, chosen a pseudo-orthonormal basis $\left\{e_{i}\right\}_{p}$ for $T_{p} M$, by means of the isometries between $p$ and any other point $p^{\prime} \in M$, one can build a pseudo-orthonormal frame field $\left\{e_{i}\right\}$ on $M$, with respect to which the components of the curvature tensor and its covariant derivatives up to order $k$ are globally constant on $M$.

In the special case when $(M, g)$ is conformally flat, this is equivalent to determining a pseudo-orthonormal frame field $\left\{e_{i}\right\}$ on $(M, g)$, such that the components of the Ricci tensor $\varrho$ and its covariant derivatives $\nabla^{i} \varrho$, for $i=1, \ldots, k$, are constant globally on $M$. To note that in particular, with respect to $\left\{e_{i}\right\}$, the components of the Ricci operator $Q$ are constant. 
Assume now that $M_{q}^{n}$ is conformally flat. Following [8], we define a tensor field $A$ of type $(1,1)$ by setting

$$
A=\frac{1}{n-2}\left(Q-\frac{S}{2(n-1)} \mathrm{Id}\right),
$$

where Id is the identity and $S$ is the scalar curvature of $(M, g)$. Then, at any point $p \in M$, $A_{p}$ is a self-adjoint linear endomorphism of the tangent space $T_{p} M$. Since $M_{q}^{n}$ is conformally flat, the equations (2.1) and (2.2) yield

$$
R(X, Y)=A X \wedge Y+X \wedge A Y, \quad \text { and } \quad\left(\nabla_{X} A\right) Y=\left(\nabla_{Y} A\right) X
$$

respectively. The following result was obtained in [8].

THEOREM 2.1 ([8]). Let $M_{q}^{n}$ be a conformally flat homogeneous pseudo-Riemannian manifold and $\lambda_{1}, \ldots, \lambda_{r}$ be the distinct eigenvalues of the tensor field $A$ on $M$ with algebraic multiplicities $m_{1}, \ldots, m_{r}$, respectively. If for $i \in\{1, \ldots, r\}$, the eigenvalue $\lambda_{i}$ is real and the dimension of its eigenspace coincides with its algebraic multiplicity, then we have

$$
\sum_{j \neq i} m_{j} \frac{\lambda_{j}+\lambda_{i}}{\lambda_{j}-\lambda_{i}}=0 .
$$

As it is well known, contrarily to the case of a definite positive inner product, a selfadjoint linear operator in a pseudo-Riemannian manifold needs not to be diagonalizable, but can assume different canonical forms. The above Theorem 2.1 was used in [8] to classify conformally flat homogeneous pseudo-Riemannian manifolds with diagonalizable Ricci operator. This classification, which does not differ essentially from the Riemannian case, is reported in the following.

THEOREM 2.2 ([8]). Let $M_{q}^{n}$ be an $n(\geq 3)$-dimensional conformally flat homogeneous pseudo-Riemannian manifold with diagonalizable Ricci operator. Then, $M_{q}^{n}$ is locally isometric to one of the following:

(i) A pseudo-Riemannian space form.

(ii) A product manifold of an m-dimensional space form of constant curvature $k \neq 0$ and an $(n-m)$-dimensional pseudo-Riemannian manifold of constant curvature $-k$, where $2 \leq m \leq n-2$.

(iii) A product manifold of an $(n-1)$-dimensional pseudo-Riemannian manifold of index $q-1$ of constant curvature $k \neq 0$ and a one-dimensional Lorentzian manifold, or a product of an $(n-1)$-dimensional pseudo-Riemannian manifold of index $q$ of constant curvature $k \neq 0$ and a one-dimensional Riemannian manifold.

Note that all examples listed in Theorem 2.2 are (locally) symmetric (in particular, Ricciparallel). Because of Theorem 2.2, we shall focus on the case when the Ricci operator is not diagonalizable. We start describing the possible canonical forms of a self-adjoint operator $A$ (equivalently, $Q$ ) on a four-dimensional conformally flat pseudo-Riemannian manifold. We obtain the following. 
THEOREM 2.3. Let $(M, g)$ denote a four-dimensional conformally flat homogeneous pseudo-Riemannian manifold.

(A) If $g$ is of signature $(2,2)$, then there exists a pseudo-orthonormal frame field $\left\{e_{1}, e_{2}, e_{3}, e_{4}\right\}$, with $e_{3}, e_{4}$ time-like vector fields, such that the self-adjoint operator $A, d e-$ fined by (2.3), takes one of the following forms:

I) The minimal polynomial of A does not admit any repeated roots:

(Ia) $\operatorname{diag}(r, \ldots,-r)$;

(Ib) $\left(\begin{array}{cccc}r & 0 & 0 & s \\ 0 & t & 0 & 0 \\ 0 & 0 & \pm t & 0 \\ s & 0 & 0 & r\end{array}\right), \begin{aligned} & s \neq 0, \\ & r^{2}+s^{2}=t^{2}\end{aligned} ; \quad$ (Ic) $\left(\begin{array}{cccc}r & 0 & s & 0 \\ 0 & t & 0 & u \\ -s & 0 & r & 0 \\ 0 & -u & 0 & t\end{array}\right), s, u \neq 0$.

II) The minimal polynomial of A has roots with multiplicity two:

(IIa) $\left(\begin{array}{cccc} \pm r & 0 & 0 & 0 \\ 0 & r+\varepsilon / 2 & -\varepsilon / 2 & 0 \\ 0 & \varepsilon / 2 & r-\varepsilon / 2 & 0 \\ 0 & 0 & 0 & \pm r\end{array}\right)$;

(IIb) $\left(\begin{array}{cccc}r+\varepsilon / 2 & 0 & -\varepsilon / 2 & 0 \\ 0 & s+\delta / 2 & 0 & -\delta / 2 \\ \varepsilon / 2 & 0 & r-\varepsilon / 2 & 0 \\ 0 & \delta / 2 & 0 & s-\delta / 2\end{array}\right)$;

(IIc) $\left(\begin{array}{cccc}r+\varepsilon / 2 & 0 & 0 & -\varepsilon / 2 \\ 0 & s & t & 0 \\ 0 & -t & s & 0 \\ \varepsilon / 2 & 0 & 0 & r-\varepsilon / 2\end{array}\right), t \neq 0$;

(IId) $\left(\begin{array}{cccc}r+1 / 2 & 0 & -1 / 2 & s \\ 0 & r-1 / 2 & -s & 1 / 2 \\ 1 / 2 & s & r-1 / 2 & 0 \\ -s & -1 / 2 & 0 & r+1 / 2\end{array}\right), s \neq 0$.

III) The minimal polynomial of A has a root with multiplicity three:

(IIIa) $\left(\begin{array}{cccc}r & \sqrt{2} / 2 & 0 & 0 \\ \sqrt{2} / 2 & r & -\sqrt{2} / 2 & 0 \\ 0 & \sqrt{2} / 2 & r & 0 \\ 0 & 0 & 0 & \pm r\end{array}\right)$; 
(IIIb) $\left(\begin{array}{cccc} \pm r & 0 & 0 & 0 \\ 0 & r & \sqrt{2} / 2 & 0 \\ 0 & -\sqrt{2} / 2 & r & \sqrt{2} / 2 \\ 0 & 0 & \sqrt{2} / 2 & r\end{array}\right)$.

IV) The minimal polynomial of A has a root with multiplicity four:

(IV) $\left(\begin{array}{cccc}r & 1 / 2 & 0 & 1 / 2 \\ 1 / 2 & r+\varepsilon / 2 & -1 / 2 & -\varepsilon / 2 \\ 0 & 1 / 2 & r & 1 / 2 \\ -1 / 2 & \varepsilon / 2 & 1 / 2 & r-\varepsilon / 2\end{array}\right)$

In the formulae above, $\varepsilon, \delta= \pm 1$.

(B) If $g$ is Lorentzian, then there exists a pseudo-orthonormal frame field $\left\{e_{1}, e_{2}, e_{3}, e_{4}\right\}$, with $e_{4}$ time-like, such that the self-adjoint operator A takes one of the following forms:

I') The minimal polynomial of A does not admit any repeated roots:

$\left(\mathrm{Ia}^{\prime}\right) \operatorname{diag}(r, \ldots,-r) ; \quad\left(\mathrm{Ib}^{\prime}\right)\left(\begin{array}{cccc}t & 0 & 0 & 0 \\ 0 & \pm t & 0 & 0 \\ 0 & 0 & r & s \\ 0 & 0 & -s & r\end{array}\right), \begin{aligned} & s \neq 0, \\ & r^{2}+s^{2}=t^{2} .\end{aligned}$

II') The minimal polynomial of A has a root with multiplicity two:

$$
\left(\mathrm{II}^{\prime}\right)\left(\begin{array}{cccc} 
\pm r & 0 & 0 & 0 \\
0 & \pm r & 0 & 0 \\
0 & 0 & r+\varepsilon / 2 & -\varepsilon / 2 \\
0 & 0 & \varepsilon / 2 & r-\varepsilon / 2
\end{array}\right)
$$

III') The minimal polynomial of A has a root with multiplicity three:

$$
\left(\mathrm{III}^{\prime}\right)\left(\begin{array}{cccc} 
\pm r & 0 & 0 & 0 \\
0 & r & \sqrt{2} / 2 & 0 \\
0 & \sqrt{2} / 2 & r & \sqrt{2} / 2 \\
0 & 0 & -\sqrt{2} / 2 & r
\end{array}\right)
$$

PROOF. According to [11], for an inner product of signature $(2,2)$ on a vector space $V$, a self-adjoint linear operator may take 10 different forms, depending on its minimal polynomial. If $A$ is diagonalizable, the equation (2.4) yields that the operator $A$ admits at most two eigenvalues $r$ and $-r$. This gives the case (Ia). 
If $A$ has a pair of complex eigenvalues $r \pm i s$, and two real eigenvalues $t \neq u$, then according to (2.4) we have

$$
\left\{\begin{array}{l}
3 t^{3}-(s+2 r) t^{2}-2 r u t+(3 u-t)\left(r^{2}+s^{2}\right), \\
3 u^{3}-(t+2 r) u^{2}-2 r t u+(3 t-u)\left(r^{2}+s^{2}\right),
\end{array}\right.
$$

whose solutions are $u= \pm t, r^{2}+s^{2}=t^{2}$. This gives the case (Ib).

If the minimal polynomial of $A$ does not admit any repeated root and has two pairs of complex eigenvalues $r \pm i s$ and $t \pm i u$, then the equation (2.4) does not give any restriction on the eigenvalues, and $A$ takes the form listed in the case (Ic).

If the minimal polynomial admits a real root $r$ with multiplicity two and two other real eigenvalues $s$ and $t$, then by (2.4) we have either $t=-s=r, t=s=-r$ or $t=s=r$. Following [11], this gives the case (IIa). The case (III) is obtained by a similar argument. For the cases (IIb), (IIc), (IId) and (IV), Theorem 2.1 does not yield any restriction, and we have the general possible form of self-adjoint operators with such properties. This ends the proof for the case of neutral signature. A similar argument applies to the case of a Lorentzian metric, for which we may also refer to [8, Theorem 2.4].

Following the standard terminology (see for example [15, Section 5.1] or [11]), given a self-adjoint operator with respect to a nondegenerate inner product, its Segre type, or Segre characteristic, lists between square brackets [ ] the sizes of Jordan blocks in the decomposition of the operator. The comma separates eigenvalues corresponding to space-like eigenvectors from those corresponding to time-like and light-like eigenvectors.

Round brackets group together different blocks referring to the same eigenvalue, while vertical lines around eigenvalues within round brackets mean that those eigenvalues are not included in the coincidence. When different blocks refer to the same eigenvalue, the Segre type is said to be degenerate. For this reason, we shall refer to the Ricci operator of a locally homogeneous pseudo-Riemannian manifold as either nondegenerate or degenerate, according to the corresponding property of its Segre type.

Because of Theorem 2.3, the possible Segre types of the Ricci operator $Q$ (equivalently, of $A$ ) for a conformally flat homogeneous four-dimensional manifold are all and the ones listed in Tables I and II.

Let now $(M, g)$ denote a pseudo-Riemannian manifold. At any point $p \in M$ and for any index $k$, consider the Lie algebra

$$
\mathfrak{g}(k, p)=\left\{Y \in \mathfrak{s o}(q, n-q) ; Y \cdot R(p)=Y . \nabla R(p)=\cdots=Y \cdot \nabla^{k} R(p)=0\right\}
$$

where $Y$ acts as a derivation. This Lie algebra measures the "isotropy" of the Riemann tensor and its first $k$ derivatives at the point $p \in M$, and is associated to the Lie subgroup $G \subset$ $S O(q, n-q)$ of linear isometries $\varphi: T_{p} M \rightarrow T_{p} M$ satisfying $\varphi^{*}\left(\nabla^{i} R(p)\right)=\nabla^{i} R(p)$ for $i=0, \ldots, k$.

Lie subalgebras $\mathfrak{g}(k, p)$ form a decreasing sequence of the Lie algebra $\mathfrak{s o}(q, n-q)$ of skew-symmetric endomorphisms of the tangent space $T_{p} M$ at $p \in M$. Thus, $\mathfrak{g}(k, p)=$ 


\begin{tabular}{|l|c|c|c|c|c|}
\hline Case & Ia & Ib & Ic & IIa & IIb \\
\hline Nondegenerate type & - & {$[1,11 \overline{1}]$} & {$[1 \overline{1} 1 \overline{1}]$} & - & {$[22]$} \\
\hline Degenerate types & {$[(11),(11)]$} & {$[(1,1) 1 \overline{1}]$} & {$[(1 \overline{1} 1 \overline{1})]$} & {$[(1,1) 2]$} & {$[(22)]$} \\
& $\begin{array}{c}{[(1|(1,1)| 1)]} \\
{[(11,1) 1]} \\
{[1(1,11)]}\end{array}$ & & & {$[1,(12)]$} & \\
& {$[(11,11)]$} & & & {$[(1,12)]$} & \\
\hline Case & IIc & IId & IIIa & IIIb & IV \\
\hline Nondegenerate type & {$[21 \overline{1}]$} & {$[2 \overline{2}]$} & {$[13]$} & {$[1,3]$} & {$[4]$} \\
\hline Degenerate types & - & - & {$[(13)]$} & {$[(1,3)]$} & - \\
\hline
\end{tabular}

TABLE I. Segre types of $Q$ for an inner product of signature $(2,2)$.

\begin{tabular}{|l|c|c|c|c|}
\hline Case & Ia & Ib & II & III \\
\hline Nondegenerate type & - & {$[11,1 \overline{1}]$} & - & {$[1,3]$} \\
\hline Degenerate types & {$[(11)(1,1)]$} & {$[(11), 1 \overline{1}]$} & {$[(11), 2]$} & {$[(1,3)]$} \\
& {$[1(11,1)]$} & & {$[1(1,2)]$} & \\
& {$[(111), 1]$} & & {$[(11,2)]$} & \\
& {$[(111,1)]$} & & & \\
\hline
\end{tabular}

TABLE II. Segre types of $Q$ for a Lorentzian inner product.

$\mathfrak{g}(k+1, p)$ for a sufficiently high $k \in N$. The smallest $k$ for which $\mathfrak{g}(k, p)=\mathfrak{g}(k+1, p)$ (at all points $p \in M$ ) is called the Singer index of $(M, g)$ and is denoted by $k_{M}$, and $\mathfrak{h}_{p}=$ $\mathfrak{g}(k, p)=\mathfrak{g}(k+1, p)$ is called the isotropy subalgebra (at $p)$. Every pseudo-Riemannian manifold $(M, g)$ which is infinitesimally homogeneous, that is, $k$-curvature homogeneous for some $k>k_{M}$, is locally homogeneous $[14,16]$. For a homogeneous pseudo-Riemannian manifold, $\mathfrak{g}(k, p)$ is isomorphic to $\mathfrak{g}\left(k, p^{\prime}\right)$ for every $p, p^{\prime} \in M$ and every non-negative integer $k$. So, in this case we simply write $g_{k}$ and $\mathfrak{h}$.

To note that if $(M, g)$ is conformally flat, by $(2.5)$ we have

$$
\mathfrak{g}(k, p)=\left\{A \in \mathfrak{s o}(q, n-q) ; A \cdot Q(p)=A \cdot \nabla Q(p)=\cdots=A \cdot \nabla^{k} Q(p)=0\right\}
$$

for any point $p \in M$ and non-negative integer $k$.

3. Cases with nondegenerate Ricci operator. In the study of conformally flat pseudo-Riemannian four-manifolds, a fundamental difference arises between the cases with nondegenerate Ricci operator $Q$ and the ones where $Q$ is degenerate. In fact, we have the following. 
THEOREM 3.1. Let $(M, g)$ be a four-dimensional conformally flat pseudo-Riemannian four-manifold. At any point $p \in M$, we have that $\mathfrak{g}(0, p)=\{0\}$ if and only if $Q_{p}$ is nondegenerate. In particular, in this case $\mathfrak{h}_{p}=0$.

PROOF. Suppose first that $\mathfrak{g}(0, p) \neq\{0\}$. Then, the Ricci operator $Q_{p}$ at $p$ must be degenerate. In fact, if $Q_{p}$ is nondegenerate, we can use the pseudo-orthonormal basis expressing $Q_{p}$ in its canonical form to prove that $\mathfrak{g}(0, p)=0$. This argument holds true for all the possible nondegenerate cases of the Ricci operator. We report below the full details for Segre type [22], the other cases are obtained by a similar argument.

So, suppose that the Ricci operator $Q_{p}$ is of nondegenerate Segre type [22]. According to Theorem 2.3, the operator $A$ has the form IIb with respect to an orthonormal frame field $\left\{e_{1}, e_{2}, e_{3}, e_{4}\right\}$, with $e_{3}, e_{4}$ time-like. By (2.3), we have $Q=(n-2) A+\operatorname{tr}(A) \operatorname{Id}$. So, setting $a=4 r+2 s$ and $b=2 r+4 s$, the Ricci operator $Q_{p}$ will take the form

$$
Q_{p}=\left(\begin{array}{cccc}
a+\varepsilon & 0 & -\varepsilon & 0 \\
0 & b+\delta & 0 & -\delta \\
\varepsilon & 0 & a-\varepsilon & 0 \\
0 & \delta & 0 & b-\delta
\end{array}\right), \quad a \neq b, \quad \varepsilon, \delta= \pm 1
$$

If $Y=\left(a_{i j}\right)$ now denotes an arbitrary element of $\mathfrak{s o}(2,2)$, we have $a_{i j}=-\varepsilon_{i} \varepsilon_{j} a_{j i}$ for all $1 \leq i, j \leq 4$. In particular, if $Y \in \mathfrak{g}(0, p)$, then we must have $Y Q_{p}\left(e_{i}\right)-Q_{p} Y\left(e_{i}\right)=0$ for all $1 \leq i \leq 4$. Using (3.7), a straightforward calculation then yields $Y=0$. Hence, $\mathfrak{g}(0, p)=0$.

Conversely, suppose now that $Q_{p}$ is degenerate. Then, for any of the canonical forms listed in Theorem 2.3, we explicitly calculated $\mathfrak{g}(0, p)$ and found $\mathfrak{g}(0, p) \neq\{0\}$. For any indices $k, h=1, \ldots, 4$, we put $E_{k h}=\left(\delta_{i k} \delta_{j h}\right)_{1 \leq i, j \leq 4}$. With respect to the same pseudoorthonormal basis $\left\{e_{1}, \ldots, e_{4}\right\}$ for which $A_{p}$ (and $Q_{p}$ ) takes its canonical form, we explicitly found:

1) Segre type $[(1,1) 1 \overline{1}]: \mathfrak{g}(0, p)=\operatorname{Span}\left(E_{23}+E_{32}\right)$.

2) Segre type [(11̄1 $\overline{1})]: \mathfrak{g}(0, p)=\operatorname{Span}\left(E_{21}-E_{12}-E_{43}+E_{34}, E_{24}-E_{13}-E_{31}+E_{42}\right)$.

3) Segre type $[(1,1) 2]: \mathfrak{g}(0, p)=\operatorname{Span}\left(E_{14}+E_{41}\right)$.

4) Segre type [1, (12)]: $\mathfrak{g}(0, p)=\operatorname{Span}\left(E_{21}-E_{12}+E_{31}+E_{13}\right)$.

5) Segre type $[(1,12)]$ : $\mathfrak{g}(0, p)=\operatorname{Span}\left(E_{21}-E_{12}+E_{13}+E_{31}, E_{14}+E_{41}, E_{43}-E_{24}-E_{34}-E_{42}\right)$.

6) Segre type $[(22)]: \mathfrak{g}(0, p)=\operatorname{Span}\left(E_{21}-E_{12}+\delta(\delta-\varepsilon)\left(E_{14}+E_{41}\right)+\varepsilon \delta\left(E_{43}-E_{34}\right)\right.$, $\left.E_{32}+E_{23}+\delta(\varepsilon+\delta)\left(E_{43}-E_{34}\right)-\varepsilon \delta\left(E_{14}+E_{41}\right)\right)$.

7) Segre type [(13)]: $\mathfrak{g}(0, p)=\operatorname{Span}\left(E_{14}+E_{41}+E_{34}-E_{43}\right)$.

8) Segre type $[(1,3)]: \mathfrak{g}(0, p)=\operatorname{Span}\left(E_{21}-E_{12}+E_{14}+E_{41}\right)$.

9) Segre type $[(11), 1 \overline{1}]: \mathfrak{g}(0, p)=\operatorname{Span}\left(E_{12}-E_{21}\right)$. 
10) Segre type [(11), 2]: $\mathfrak{g}(0, p)=\operatorname{Span}\left(E_{12}-E_{21}\right)$.

11) Segre type $[1(1,2)]: \mathfrak{g}(0, p)=\operatorname{Span}\left(E_{24}-E_{23}+E_{32}+E_{42}\right)$.

12) Segre type $[(11,2)]: \mathfrak{g}(0, p)=\operatorname{Span}\left(E_{21}-E_{12}, E_{31}+E_{41}-E_{13}+E_{14}\right.$, $\left.E_{32}+E_{42}-E_{23}+E_{24}\right)$.

13) Lorentzian Segre type $[(1,3)]: \mathfrak{g}(0, p)=\operatorname{Span}\left(E_{12}+E_{14}-E_{21}+E_{41}\right)$.

Finally, we remark that one finds $\mathfrak{g}_{0} \neq\{0\}$ also for degenerate types with a diagonalizable Ricci operator. However, since the classification for those types has been already given in [8], we did not report the corresponding algebra $\mathfrak{g}(0, p)$ here.

If $(M, g)$ is a four-dimensional conformally flat homogeneous pseudo-Riemannian manifold, then the Segre type of its Ricci operator is the same at any point, and Theorem 3.1 yields at once the following.

Corollary 3.2. Let $(M, g)$ be a four-dimensional conformally flat homogeneous pseudo-Riemannian four-manifold. If the Ricci operator $Q$ of $(M, g)$ is nondegenerate, then $(M, g)$ is locally isometric to a Lie group equipped with a left-invariant pseudo-Riemannian metric.

Taking into account the above result, we shall now classify conformally flat homogeneous pseudo-Riemannian four-manifolds, in the cases where $Q$ is nondegenerate. We start proving the following result.

THEOREM 3.3. Let $(M, g)$ be a conformally flat homogeneous pseudo-Riemannian four-manifold. If the Ricci operator $Q$ of $(M, g)$ is not diagonalizable and nondegenerate, then $Q$ can only be of Segre type $[1,11 \overline{1}]$ if $g$ is neutral, or $[11,1 \overline{1}]$ if $g$ is Lorentzian.

PROOF. This result is obtained from a case-by-case argument, starting from the possible nondegenerate Segre types of tensor $A$, as classified in Theorem 2.3.

If $A$ is of nondegenerate Segre type [22], then, according to Theorem 2.3, there exists a pseudo-orthonormal frame field $\left\{e_{i}\right\}$, with respect to which $A$ takes the form IIb with $r \neq s$. As explained in the proof of Theorem 3.1, the Ricci operator will be then described by (3.7) (at any point $p$ ) with respect to the frame field $\left\{e_{i}\right\}$.

By Corollary 3.2, $(M, g)$ is locally isometric to a Lie group with a left-invariant metric. Let $\left\{e_{1}, \ldots, e_{4}\right\}$ denote a left-invariant orthonormal frame field. The Levi-Civita connection is then completely described by $\nabla_{e_{i}} e_{j}=\Gamma_{i j}^{k} e_{k}$, where $\Gamma_{i j}^{k}$ are some real constants, satisfying

$$
\Gamma_{i j}^{k}=-\varepsilon_{j} \varepsilon_{k} \Gamma_{i k}^{j},
$$

for all indices $i, j, k$. Using (3.7) to calculate $\nabla_{e_{k}} Q$, we find

$$
\nabla_{e_{k}} Q=\left(\begin{array}{cccc}
2 \Gamma_{11}^{3} \varepsilon & \phi_{k} & -2 \Gamma_{k 1}^{3} \varepsilon & \psi_{k} \\
\phi_{k} & 2 \Gamma_{k 2}^{4} \delta & -\theta_{k} & -2 \Gamma_{k 2}^{4} \delta \\
2 \Gamma_{k 1}^{3} \varepsilon & \theta_{k} & -2 \Gamma_{k 1}^{3} \varepsilon & \eta_{k} \\
-\psi_{k} & 2 \Gamma_{k 2}^{4} \delta & \eta_{k} & -2 \Gamma_{k 2}^{4} \delta
\end{array}\right), \quad k=1, \ldots, 4,
$$


where we put

$$
\begin{array}{ll}
\phi_{k}=\Gamma_{k 1}^{2}(a-b+\varepsilon-\delta)+\Gamma_{k 1}^{4} \delta+\Gamma_{k 2}^{3} \varepsilon, & \psi_{k}=\Gamma_{k 1}^{2} \delta+\Gamma_{k 1}^{4}(b-a-\delta-\varepsilon)-\Gamma_{k 3}^{4} \varepsilon, \\
\theta_{k}=\Gamma_{k 2}^{3}(b-a+\delta+\varepsilon)-\Gamma_{k 3}^{4} \delta+\Gamma_{k 1}^{2} \varepsilon, & \eta_{k}=-\Gamma_{k 2}^{3} \delta-\Gamma_{k 3}^{4}(a+b+\delta-\varepsilon)-\Gamma_{k 1}^{4} \varepsilon .
\end{array}
$$

Since $(M, g)$ is homogeneous and conformally flat, we must have

$$
\left(\nabla_{X} Q\right)(Y)=\left(\nabla_{Y} Q\right)(X)
$$

for all tangent vector fields $X, Y$. Denoting by $\Gamma_{k}$ the matrix $\left(\Gamma_{k}\right)_{i j}=\Gamma_{k j}^{i}$, for all indices $i, j, k$, we apply the equation (3.9) and determine the Levi-Civita coefficients as follows:

$$
\begin{aligned}
& \Gamma_{1}=\left(\begin{array}{cccc}
0 & \frac{-2 \alpha \varepsilon+\eta(a-b+\delta)}{\delta} & -\gamma & -\eta \\
\frac{2 \alpha \varepsilon-\eta(a+b-\delta)}{\delta} & 0 & \frac{-2 \alpha \varepsilon+\eta(a-b+\delta)}{\delta} & -\frac{\beta(a-b+\varepsilon)+\nu \varepsilon}{2 \delta} \\
-\gamma & \frac{-2 \alpha \varepsilon+\eta(a-b+\delta)}{\delta} & 0 & -\eta \\
-\eta & -\frac{\beta(a-b+\varepsilon)+\nu \varepsilon}{2 \delta} & \beta & 0
\end{array}\right), \\
& \Gamma_{2}=\left(\begin{array}{cccc}
0 & \beta & -\frac{2 \alpha \varepsilon(b-a)+\eta(b-a)^{2}+2 \delta \alpha \varepsilon}{2 \delta \varepsilon} & -\beta \\
-\beta & 0 & -v & -\mu \\
-\frac{2 \alpha \varepsilon(b-a)+\beta(b-a)^{2}+2 \delta \alpha \varepsilon}{2 \delta \varepsilon} & -v & 0 & v \\
-\beta & -\mu & -v & 0
\end{array}\right), \\
& \Gamma_{3}=\left(\begin{array}{cccc}
0 & -\frac{-2 \alpha \varepsilon+\eta(a-b+\delta)}{\delta} & \gamma & \eta \\
\frac{-2 \alpha \varepsilon+\eta(a-b+\delta)}{\delta} & 0 & -\frac{-2 \alpha \varepsilon+\eta(a-b+\delta)}{\delta} & -\frac{\nu(a-b-\varepsilon)-\beta \varepsilon}{2 \delta} \\
\gamma & -\frac{-2 \alpha \varepsilon+\eta(a-b+\delta)}{\delta} & 0 & \eta \\
\eta & -\frac{\nu(a-b-\varepsilon)-\beta \varepsilon}{2 \delta} & -\beta & 0
\end{array}\right), \\
& \Gamma_{4}=\left(\begin{array}{cccc}
0 & -\beta & \alpha & \beta \\
\beta & 0 & v & \mu \\
\alpha & v & 0 & -v \\
\beta & \mu & v & 0
\end{array}\right),
\end{aligned}
$$

where we put $\alpha=\Gamma_{41}^{3}, \beta=\Gamma_{41}^{4}, \gamma=\Gamma_{31}^{3}, \mu=\Gamma_{42}^{4}, \eta=\Gamma_{31}^{4}$ and $v=\Gamma_{43}^{4}$. We can now calculate the curvature of $(M, g)$ in terms of $\Gamma_{i j}^{k}$. In particular, with respect to $\left\{e_{i}\right\}$, a standard calculation yields

$$
\begin{aligned}
& R\left(e_{1}, e_{3}\right) e_{1}=-(1 / 2 \delta)(v+\beta)(a-b)\left(\eta e_{2}+\delta(-2 \alpha \varepsilon+\eta(a-b+\delta)) e_{4}\right), \\
& R\left(e_{2}, e_{4}\right) e_{2}=(1 / 2 \varepsilon \delta)(a-b)(-2 \alpha \varepsilon+\eta(a-b))\left(-v e_{1}+\beta e_{3}\right) .
\end{aligned}
$$

On the other hand, since $(M, g)$ is conformally flat, by (3.7) we obtain

$$
R\left(e_{1}, e_{3}\right) e_{1}=(1 / 3)(b-2 a) e_{3}, \quad R\left(e_{2}, e_{4}\right) e_{2}=(1 / 3)(a-2 b) e_{4} .
$$


Comparison between the above equations for $R\left(e_{1}, e_{3}\right) e_{1}$ and $R\left(e_{2}, e_{4}\right) e_{2}$, easily permits to conclude that $a=b=0$, which contradicts the Segre type of $Q$. Thus, this case cannot occur. By similar argument and calculations, we found that none of nondegenerate Segre types [1 $1 \overline{1} \overline{1}],[21 \overline{1}],[2 \overline{2}],[13],[1,3]$ and [4] can occur for a four-dimensional conformally flat pseudo-Riemannian manifold of neutral signature, and that nondegenerate Segre type $[1,3]$ cannot occur in Lorentzian settings.

We now completely describe four-dimensional conformally flat pseudo-Riemannian Lie groups of neutral signature, whose Ricci operator is of nondegenerate Segre type [1, 111] ].

THEOREM 3.4. Let $(M, g)$ be a conformally flat homogeneous four-dimensional manifold with the Ricci operator of Segre type $[1,11 \overline{1}]$. Then, $(M, g)$ is locally isometric to one of the following unsolvable Lie groups:

(i) Either $S U(2) \times \boldsymbol{R}($ when $\varepsilon=1)$ or $S L(2, \boldsymbol{R}) \times \boldsymbol{R}$ (when $\varepsilon=-1)$, equipped with a left-invariant neutral metric, admitting a pseudo-orthonormal basis $\left\{e_{1}, e_{2}, e_{3}, e_{4}\right\}$ for the Lie algebra, such that the Lie brackets take the form

$$
\begin{aligned}
& {\left[e_{1}, e_{2}\right]=\varepsilon \alpha e_{3}, \quad\left[e_{1}, e_{3}\right]=-\varepsilon \alpha e_{2}, \quad\left[e_{2}, e_{3}\right]=2 \alpha\left(e_{1}+\varepsilon e_{4}\right),} \\
& {\left[e_{2}, e_{4}\right]=-\alpha e_{3}, \quad\left[e_{3}, e_{4}\right]=\alpha e_{2},}
\end{aligned}
$$

(ii) $S L(2, \boldsymbol{R}) \times \boldsymbol{R}$, equipped with a left-invariant neutral metric, admitting a pseudoorthonormal basis $\left\{e_{1}, e_{2}, e_{3}, e_{4}\right\}$ for the Lie algebra, such that the Lie brackets take the form

$$
\begin{array}{ll}
{\left[e_{1}, e_{2}\right]=-\varepsilon \alpha e_{1},} & {\left[e_{1}, e_{3}\right]=\alpha e_{1}, \quad\left[e_{1}, e_{4}\right]=2 \alpha\left(\varepsilon e_{2}-e_{3}\right),} \\
{\left[e_{2}, e_{4}\right]=-\varepsilon \alpha e_{4},} & {\left[e_{3}, e_{4}\right]=\alpha e_{4},}
\end{array}
$$

where $\alpha \neq 0$ is a real constant and $\varepsilon= \pm 1$.

PROOF. We already know from Corollary 3.2 that $(M, g)$ is locally isometric to some Lie group $G$ with a left-invariant metric. Consider a pseudo-orthonormal basis $\left\{e_{1}, \ldots, e_{4}\right\}$ of the Lie algebra $\mathfrak{g}$ of $G$, with respect to which the operator $A$ takes the form (Ib). Then, by (2.3) we have $Q=(n-2) A+\operatorname{tr}(A)$ Id. We set $a=4 r, b=2 s$ and $c=2(r+t)$. With respect to $\left\{e_{i}\right\}$, the Ricci operator is then given by

$$
Q=\left(\begin{array}{cccc}
a & 0 & 0 & b \\
0 & c & 0 & 0 \\
0 & 0 & -c & 0 \\
-b & 0 & 0 & a
\end{array}\right) \quad \text { with } b^{2}=c^{2}-a c \neq 0
$$

Note that $b^{2}=c^{2}-a c \neq 0$ implies $c \neq 0$ and so, $a=\left(c^{2}-b^{2}\right) / c$.

The Levi-Civita connection is described by $\nabla_{e_{i}} e_{j}=\Gamma_{i j}^{k} e_{k}$ for some real constants $\Gamma_{i j}^{k}$ satisfying (3.8). Proceeding as in the proof of Theorem 3.3, we first calculate $\nabla_{e_{k}} Q$ and apply (3.9) to express all coefficients $\Gamma_{i j}^{k}$ in function of $\beta=\Gamma_{23}^{4}, \gamma=\Gamma_{32}^{4}, \Gamma_{41}^{3}=\theta$ and $\Gamma_{42}^{4}=\eta$. 
Explicitly, we find

$$
\begin{aligned}
& \Gamma_{1}=\left(\begin{array}{cccc}
0 & -\eta & -\frac{\theta\left(b^{2}-2 c^{2}\right)}{b c} & 0 \\
\eta & 0 & \frac{c^{4}(2 \gamma+4 \beta)+b^{4}(\beta-\gamma)+b^{2} c^{2}(\gamma-3 \beta)}{4 c^{5}} & \frac{\eta c}{b} \\
-\frac{\theta\left(b^{2}-2 c^{2}\right)}{b c} & \frac{c^{4}(2 \gamma+4 \beta)+b^{4}(\beta-\gamma)+b^{2} c^{2}(\gamma-3 \beta)}{4 c^{5}} & 0 & -\theta \\
0 & \frac{\eta c}{b} & \alpha & 0
\end{array}\right), \\
& \Gamma_{2}=\left(\begin{array}{cccc}
0 & 0 & -\frac{b\left(b^{2}(\beta-\gamma)-c^{2}(\beta-\gamma)\right)}{2 c^{3}} & \frac{\eta\left(b^{2}+c^{2}\right)}{2 b c} \\
0 & 0 & 0 & 0 \\
-\frac{b\left(b^{2}(\beta-\gamma)-c^{2}(\beta-\gamma)\right)}{2 c^{3}} & 0 & 0 & -\beta \\
\frac{\eta\left(b^{2}+c^{2}\right)}{2 b c} & 0 & \beta & 0
\end{array}\right) \\
& \Gamma_{3}=\left(\begin{array}{cccc}
0 & -\frac{4 \beta c^{2}+b^{4}(\beta-\gamma)+b^{2} c^{2}(\gamma-3 \beta)}{2 c^{3} b} & 0 & \frac{\theta\left(b^{4}-3 b^{2} c^{2}+4 c^{4}\right)}{2 b^{2} c^{2}} \\
\frac{4 \beta c^{2}+b^{4}(\beta-\gamma)+b^{2} c^{2}(\gamma-3 \beta)}{2 c^{3} b} & 0 & 0 & \gamma \\
0 & 0 & 0 & 0 \\
\frac{\theta\left(b^{4}-3 b^{2} c^{2}+4 c^{4}\right)}{2 b^{2} c^{2}} & \gamma & 0 & 0
\end{array}\right), \\
& \Gamma_{4}=\left(\begin{array}{cccc}
0 & \frac{\eta c}{b} & \theta & 0 \\
-\frac{\eta c}{b} & 0 & -\frac{4 \beta c^{4}+b^{4}(\beta-\gamma)-b^{2} c^{2}(\gamma+3 \beta)}{4 c^{4}} & \eta \\
\theta & -\frac{4 \beta c^{4}+b^{4}(\beta-\gamma)-b^{2} c^{2}(\gamma+3 \beta)}{4 c^{4}} & 0 & -\frac{\theta\left(b^{2}-2 c^{2}\right)}{b c} \\
0 & \eta & \frac{\theta\left(b^{2}-2 c^{2}\right)}{b c} & 0
\end{array}\right) .
\end{aligned}
$$

We now calculate the curvature tensor of $(M, g)$ with respect to $\left\{e_{i}\right\}$. Using the above description of the Levi-Civita connection, a direct calculation yields that the scalar curvature vanishes. On the other hand, by (3.10) we have that the scalar curvature is given by $2\left(c^{2}-b^{2}\right) / c$. Therefore, $c= \pm b$. Taking into account this condition, the components of the Ricci operator with respect to $\left\{e_{i}\right\}$ are given by

$$
\begin{aligned}
& Q_{11}=2\left(\eta^{2}-\beta \gamma-\theta^{2}\right), \quad Q_{12}=\frac{3}{2} \theta(\beta-\gamma), \quad Q_{13}= \pm \frac{3}{2} \eta(\beta-\gamma), \\
& Q_{14}= \pm\left(-2 \eta^{2}-2 \theta^{2}+\beta^{2}-\gamma^{2}\right), Q_{22}=2 \gamma \beta-4 \eta^{2}+\beta^{2}-\gamma^{2}, Q_{23}=0 \\
& Q_{24}=\mp \frac{3}{2} \theta(\gamma+\beta), \quad Q_{33}=2 \gamma \beta-\beta^{2}+\gamma^{2}+4 \theta^{2}, Q_{34}=\frac{3}{2} \eta(\gamma+\beta), \\
& Q_{44}=2\left(\eta^{2}-\beta \gamma-\theta^{2}\right) \text {, }
\end{aligned}
$$

which, compared with (3.10), yield that the coefficients $\Gamma_{i j}^{k}$ satisfy one of the following sets of conditions:

$$
\text { either } \eta=\gamma=\theta=0, \quad \eta=\beta=\theta=0 \quad \text { or } \quad \beta=\gamma=\eta \pm \theta=0 \text {. }
$$


Consequently, with respect to the pseudo-orthonormal basis $\left\{e_{i}\right\}$, the Lie brackets are completely described either by

$$
\begin{aligned}
& {\left[e_{1}, e_{2}\right]=\varepsilon \alpha e_{3}, \quad\left[e_{1}, e_{3}\right]=-\varepsilon \alpha e_{2}, \quad\left[e_{2}, e_{3}\right]=2 \alpha\left(e_{1}+\varepsilon e_{4}\right),} \\
& {\left[e_{2}, e_{4}\right]=-\alpha e_{3}, \quad\left[e_{3}, e_{4}\right]=\alpha e_{2}}
\end{aligned}
$$

(for the first two sets of the above conditions), or by

$$
\begin{aligned}
& {\left[e_{1}, e_{2}\right]=-\varepsilon \alpha e_{1}, \quad\left[e_{1}, e_{3}\right]=\alpha e_{1}, \quad\left[e_{1}, e_{4}\right]=2 \alpha\left(\varepsilon e_{2}-e_{3}\right),} \\
& {\left[e_{2}, e_{4}\right]=-\varepsilon \alpha e_{4}, \quad\left[e_{3}, e_{4}\right]=\alpha e_{4}}
\end{aligned}
$$

(for the remaining set of conditions), where $\alpha \neq 0$ is a real constant and $\varepsilon= \pm 1$.

Finally, for the first of the two cases listed above, we consider the new basis of $\mathfrak{g}$ given by $\left\{\hat{e}_{1}=e_{1}+\varepsilon e_{4}, \hat{e}_{2}=e_{2}, \hat{e}_{3}=e_{3}, \hat{e}_{4}=e_{1}-\varepsilon e_{4}\right\}$, and we see that the non-zero Lie brackets are given by

$$
\left[\hat{e}_{1}, \hat{e}_{2}\right]=2 \varepsilon \alpha \hat{e}_{3}, \quad\left[\hat{e}_{1}, \hat{e}_{3}\right]=-2 \varepsilon \alpha \hat{e}_{2}, \quad\left[\hat{e}_{2}, \hat{e}_{3}\right]=2 \alpha \hat{e}_{1} .
$$

In the same way, setting $\left\{\hat{e}_{1}=e_{1}, \hat{e}_{2}=e_{2}-\varepsilon e_{3}, \hat{e}_{3}=e_{4}, \hat{e}_{4}=e_{2}+\varepsilon e_{3}\right\}$, the non-zero Lie brackets for the second case are

$$
\left[\hat{e}_{1}, \hat{e}_{2}\right]=-2 \varepsilon \alpha \hat{e}_{1}, \quad\left[\hat{e}_{1}, \hat{e}_{3}\right]=2 \varepsilon \alpha \hat{e}_{2}, \quad\left[\hat{e}_{2}, \hat{e}_{3}\right]=-2 \varepsilon \alpha \hat{e}_{3} .
$$

Thus, we conclude that in both cases the four-dimensional Lie algebra is the direct sum of a one-dimensional algebra $\mathfrak{r}$ and a three-dimensional unsolvable Lie algebra. More precisely, from the classification of three-dimensional real Lie algebras (see for example [1]), we conclude that in the first case, $G$ is locally isometric to one of the direct products $S U(2) \times \boldsymbol{R}$ or $S L(2, \boldsymbol{R}) \times \boldsymbol{R}$, depending on the value of $\varepsilon$, while in the second case, $G$ is $S L(2, \boldsymbol{R}) \times \boldsymbol{R}$, for both values of $\varepsilon$.

By a similar argument we obtained the Lorentzian analogue of the above result, given by the following.

THEOREM 3.5. Let $(M, g)$ be a conformally flat homogeneous Lorentzian fourmanifold with the Ricci operator of Segre type $[11,1 \overline{1}]$. Then, $(M, g)$ is locally isometric to the unsolvable Lie group $S L(2, \boldsymbol{R}) \times \boldsymbol{R}$, equipped with a left-invariant Lorentzian metric, admitting a pseudo-orthonormal basis $\left\{e_{1}, e_{2}, e_{3}, e_{4}\right\}$ for the Lie algebra, such that the Lie brackets take one of the forms

$$
\begin{aligned}
& \text { (i) }\left[e_{1}, e_{2}\right]=-2 \alpha\left(\varepsilon e_{3}+e_{4}\right), \quad\left[e_{1}, e_{3}\right]=\varepsilon \alpha e_{2}, \quad\left[e_{1}, e_{4}\right]=\alpha e_{2} \text {, } \\
& {\left[e_{2}, e_{3}\right]=\varepsilon \alpha e_{1}, \quad\left[e_{2}, e_{4}\right]=\alpha e_{1},} \\
& \text { (ii) }\left[e_{1}, e_{2}\right]=2 \alpha\left(\varepsilon e_{3}+e_{4}\right), \quad\left[e_{1}, e_{3}\right]=\varepsilon \alpha e_{2}, \quad\left[e_{1}, e_{4}\right]=\alpha e_{2} \text {, } \\
& {\left[e_{2}, e_{3}\right]=\varepsilon \alpha e_{1}, \quad\left[e_{2}, e_{4}\right]=\alpha e_{1},}
\end{aligned}
$$

where $\alpha \neq 0$ is a real constant and $\varepsilon= \pm 1$. For the first Lie algebra, considering the new basis $\left\{\hat{e}_{1}=e_{1}, \hat{e}_{2}=e_{2}, \hat{e}_{3}=e_{3}+\varepsilon e_{4}, \hat{e}_{4}=e_{3}-\varepsilon e_{4}\right\}$ for $\mathfrak{g}$, the nonvanishing Lie brackets are

$$
\left[\hat{e}_{1}, \hat{e}_{2}\right]=-2 \varepsilon \alpha \hat{e}_{3}, \quad\left[\hat{e}_{1}, \hat{e}_{3}\right]=2 \varepsilon \alpha \hat{e}_{2}, \quad\left[\hat{e}_{2}, \hat{e}_{3}\right]=2 \varepsilon \alpha \hat{e}_{1}
$$


Similarly, with respect to the basis $\left\{\hat{e}_{1}=e_{1}, \hat{e}_{2}=e_{2}, \hat{e}_{3}=e_{3}+\varepsilon e_{4}, \hat{e}_{4}=e_{3}-\varepsilon e_{4}\right\}$, the nonvanishing Lie brackets for the second Lie algebra are

$$
\left[\hat{e}_{1}, \hat{e}_{2}\right]=2 \varepsilon \alpha \hat{e}_{3}, \quad\left[\hat{e}_{1}, \hat{e}_{3}\right]=2 \varepsilon \alpha e_{2}, \quad\left[\hat{e}_{2}, \hat{e}_{3}\right]=2 \varepsilon \alpha \hat{e}_{1} .
$$

4. Cases with degenerate Ricci operator and trivial isotropy. We are left to determine conformally flat homogeneous pseudo-Riemannian four-manifolds with a Ricci operator of degenerate Segre type.

We first consider the Ricci-parallel examples. Note that if a four-dimensional homogeneous pseudo-Riemannian manifold $(M, g)$ is Ricci-parallel, then its Ricci operator $Q$ is necessarily degenerate [4], [6]. Moreover, it is well known that a Ricci-parallel conformally flat manifold is locally symmetric.

If $Q$ is diagonalizable, we may refer to Theorem 2.2 for the complete classification of Ricci-parallel examples in any dimension greater than or equal to three. On the other hand, Ricci-parallel homogeneous pseudo-Riemannian four-manifolds have been investigated in [4] for the Lorentzian case and in [6] for the neutral signature case. Finally, not only locally symmetric examples, but all conformally flat (simply connected, complete) pseudo-Riemannian manifolds satisfying the weaker condition $R(X, Y) \cdot Q=0$ have been completely described in [7]. Sorting out the conformally flat Ricci-parallel (hence, locally symmetric) examples in the classification given in the Main theorem of [7] and in [4], [6], we get the following.

PROPOSITION 4.1. Let $(M, g)$ be a conformally flat Ricci-parallel homogeneous pseudo-Riemannian four-manifold and $Q$ its Ricci operator.

(i) If $Q$ is diagonalizable, then $(M, g)$ is locally isometric to one of the spaces (of dimension $n=4$ ) listed in Theorem 2.2.

(ii) If $Q$ is not diagonalizable, then either

(a) $(M, g)$ is locally isometric to a complex sphere in $C^{3}$ [7], defined by

$$
z_{1}^{2}+z_{2}^{2}+z_{3}^{2}=i b \quad(b \neq 0, b \in \boldsymbol{R}), \quad \text { or }
$$

(b) $(M, g)$ is a (conformally flat, locally symmetric) Walker manifold. In this case, $Q$ is two-step nilpotent, that is, $Q^{2}=0$.

For the description of four-dimensional Walker manifolds, we may refer to [2], [5] and references therein. With regard to which Segre types of the Ricci operator allow the existence of conformally flat Ricci-parallel homogeneous four-manifolds, the above Proposition 4.1 yields the following.

COROLlary 4.2. Conformally flat Ricci-parallel homogeneous pseudo-Riemannian four-manifolds only occur for the following degenerate Segre types of the Ricci operator:

(a) When $g$ is of neutral signature: [(11), (11)], [(11, 1)1], [1(1,11)], [(11,11)] if $Q$ is diagonalizable; $[(1 \overline{1} 1 \overline{1})],[(1,12)]$ and $[(22)]$ if $Q$ is not diagonalizable.

(b) When $g$ is Lorentzian: $[(11)(1,1)],[(111), 1],[1(11,1)],[(111,1)]$ if $Q$ is diagonalizable; $[(11,2)]$ if $Q$ is not diagonalizable. 
Proposition 4.1 leaves us to consider conformally flat pseudo-Riemannian homogeneous four-manifolds, with Ricci operator of a degenerate Segre type, which are not locally symmetric. Indeed, what makes particularly interesting to determine conformally flat (locally) homogeneous pseudo-Riemannian manifolds is the fact that, contrarily to the Riemannian case [17], they need not to be (locally) symmetric.

So, let now $(M, g)$ denote a four-dimensional conformally flat homogeneous pseudoRiemannian manifold, not Ricci-parallel, with degenerate Ricci operator. Then, Theorem 3.1 yields that $g_{0} \neq 0$, but this is not sufficient to conclude that the isotropy subalgebra $\mathfrak{h}$ does not vanish. Indeed, to study four-dimensional conformally flat homogeneous pseudo-Riemannian manifold with degenerate Ricci operator, we shall consider two distinct cases, according to whether $\mathfrak{h} \neq 0$ or not. The rest of this Section is devoted to the case when $\mathfrak{h}=0$.

If $\mathfrak{h}=0$, then $(M, g)$ is locally isometric to some four-dimensional Lie group, equipped with a left-invariant conformally flat pseudo-Riemannian metric. We start considering metrics of neutral signature, for which the possible cases are listed in the following.

THEOREM 4.3. Let $(M, g)$ be a conformally flat, not Ricci-parallel, homogeneous four-manifold of neutral signature, with a degenerate and not diagonalizable Ricci operator Q. If $(M, g)$ has trivial isotropy, then $(M, g)$ is locally isometric to a Lie group $G$, equipped with a left-invariant neutral metric, and $Q$ is of one of the Segre types [1, $(12)],[(1,12)]$, [(22)], [(13)] or [(1,3)]. More precisely, we list below an explicit description of the Lie algebra $\mathfrak{g}$ of $G$, with respect to a pseudo-orthonormal basis $\left\{e_{1}, e_{2}, e_{3}, e_{4}\right\}$, with $e_{3}, e_{4}$ time-like:

(1) Segre type [1, (12)]: $G$ is the solvable Lie group $=\boldsymbol{R} \ltimes E(1,1)$, whose Lie algebra $\mathfrak{g}$ is described by

$$
\begin{aligned}
& {\left[e_{1}, e_{2}\right]=-c_{1} e_{1}+\varepsilon \frac{\sqrt{2}\left(4 c_{1}^{2}+4 c_{2}^{2}-1\right)}{4 c_{2}} e_{2}+\varepsilon \frac{\sqrt{2}\left(4 c_{1}^{2}+2 c_{2}^{2}-1\right)}{4 c_{2}} e_{3},} \\
& {\left[e_{1}, e_{3}\right]=c_{1} e_{1}-\varepsilon \frac{\sqrt{2}\left(4 c_{1}^{2}-2 c_{2}^{2}-1\right)}{4 c_{2}} e_{2}-\varepsilon \frac{\sqrt{2}\left(4 c_{1}^{2}-4 c_{2}^{2}-1\right)}{4 c_{2}} e_{3},} \\
& {\left[e_{1}, e_{4}\right]=\delta c_{1} e_{2}+\delta c_{1} e_{3}, \quad\left[e_{2}, e_{3}\right]=3 c_{1} e_{2}+3 c_{1} e_{3},} \\
& {\left[e_{2}, e_{4}\right]=-\delta c_{1} e_{1}+\delta \varepsilon \frac{\sqrt{2}}{4 c_{2}} e_{2}+\delta \varepsilon \frac{\sqrt{2}\left(1-2 c_{2}^{2}\right)}{4 c_{2}} e_{3},} \\
& {\left[e_{3}, e_{4}\right]=\delta c_{1} e_{1}-\delta \varepsilon \frac{\sqrt{2}\left(1+2 c_{2}^{2}\right)}{4 c_{2}} e_{2}-\delta \varepsilon \frac{\sqrt{2}}{4 c_{2}} e_{3},}
\end{aligned}
$$

for any real constants $c_{1}, c_{2} \neq 0$.

(2) Segre type $[(1,12)]$ : An explicit solution is given by the solvable Lie group $G=$ $\boldsymbol{R} \ltimes \boldsymbol{R}^{3}$, whose Lie algebra $\mathfrak{g}$ is described by

$$
\begin{aligned}
& {\left[e_{1}, e_{2}\right]=-\left[e_{1}, e_{3}\right]=-\frac{1}{2 c_{1}} e_{1}-c_{2} e_{2}-c_{2} e_{3}, \quad\left[e_{2}, e_{3}\right]=\frac{2 c_{1}^{2}+1}{2 c_{1}} e_{2}+\frac{2 c_{1}^{2}+1}{2 c_{1}} e_{3},} \\
& {\left[e_{2}, e_{4}\right]=-\left[e_{3}, e_{4}\right]=c_{3} e_{2}+c_{3} e_{3}+c_{1} e_{4},}
\end{aligned}
$$


for any real constants $c_{1} \neq 0, c_{2}, c_{3}$.

(3) Segre type [(22)]: An explicit solution is given by the solvable Lie group $G=$ $\boldsymbol{R} \ltimes H$ (where $H$ denotes the Heisenberg group), whose Lie algebra $\mathfrak{g}$ is described by

$$
\begin{array}{ll}
{\left[e_{1}, e_{2}\right]=\frac{1-4 c_{1}^{2}}{4 c_{1}} e_{2}+\frac{1}{8 c_{1}} e_{4},} & {\left[e_{1}, e_{3}\right]=\frac{1+8 c_{1}^{2}}{4 c_{1}} e_{1}+\frac{1+8 c_{1}^{2}}{4 c_{1}} e_{3},} \\
{\left[e_{1}, e_{4}\right]=\frac{1+16 c_{1}^{2}}{8 c_{1}} e_{2}+\frac{1+4 c_{1}^{2}}{4 c_{1}} e_{4},} & {\left[e_{2}, e_{3}\right]=\frac{1+4 c_{1}^{2}}{4 c_{1}} e_{2}+\frac{1+16 c_{1}^{2}}{8 c_{1}} e_{4},} \\
{\left[e_{3}, e_{4}\right]=-\frac{1}{8 c_{1}} e_{2}-\frac{1-4 c_{1}^{2}}{4 c_{1}} e_{4},} &
\end{array}
$$

for any real constant $c_{1} \neq 0$.

(4) Segre type [(13)]: An explicit solution is given by the solvable Lie group $G=$ $\boldsymbol{R} \ltimes E(1,1)$, whose Lie algebra $\mathfrak{g}$ is described by

$$
\begin{array}{lll}
{\left[e_{1}, e_{2}\right]=\frac{3}{4 c_{1}^{3}} e_{1}+\frac{\sqrt{2}}{2 c_{1}} e_{2}+\frac{3}{4 c_{1}^{3}} e_{3},} & {\left[e_{1}, e_{3}\right]=\frac{\sqrt{2}}{c_{1}} e_{1}+\frac{\sqrt{2}}{c_{1}} e_{3},} \\
{\left[e_{1}, e_{4}\right]=-c_{1} e_{1}-\frac{\sqrt{2}}{4 c_{1}} e_{2}-c_{1} e_{3},} & {\left[e_{2}, e_{3}\right]=\frac{3}{4 c_{1}^{3}} e_{1}+\frac{\sqrt{2}}{2 c_{1}} e_{2}+\frac{3}{4 c_{1}^{3}} e_{3},} \\
{\left[e_{2}, e_{4}\right]=-\frac{3 \sqrt{2}}{4 c_{1}} e_{1}-c_{1} e_{2}-\frac{3 \sqrt{2}}{4 c_{1}} e_{3},} & {\left[e_{3}, e_{4}\right]=-c_{1} e_{1}+\frac{\sqrt{2}}{4 c_{1}} e_{2}-c_{1} e_{3},}
\end{array}
$$

for any real constant $c_{1} \neq 0$.

(5) Segre type [(1,3)]: $G$ is the solvable Lie group $=\boldsymbol{R} \ltimes E(1,1)$, whose Lie algebra $\mathfrak{g}$ is described by

$$
\begin{aligned}
& {\left[e_{1}, e_{2}\right]=\left(c_{1}-c_{2}\right) e_{2}-\frac{\sqrt{2}}{4 c_{2}} e_{3}+\left(c_{1}-c_{2}\right) e_{4}, \quad\left[e_{1}, e_{3}\right]=\frac{3 \sqrt{2}}{4 c_{2}} e_{2}-c_{2} e_{3}+\frac{3 \sqrt{2}}{4 c_{2}} e_{4},} \\
& {\left[e_{1}, e_{4}\right]=-\left(c_{1}+c_{2}\right) e_{2}+\frac{\sqrt{2}}{4 c_{2}} e_{3}-\left(c_{1}+c_{2}\right) e_{4}, \quad\left[e_{2}, e_{4}\right]=\sqrt{2} \phi c_{2}\left(e_{2}+e_{4}\right),} \\
& {\left[e_{2}, e_{3}\right]=\left[e_{3}, e_{4}\right]=-\frac{3 \phi}{4 c_{2}} e_{2}+\frac{\sqrt{2} \phi c_{2}}{2} e_{3}-\frac{3 \phi}{4 c_{2}} e_{4},}
\end{aligned}
$$

where $\phi= \pm \sqrt{1-2 c_{1} c_{2}^{3}} / c_{2}^{2}$, for any real constants $c_{1}, c_{2} \neq 0$ such that $1-2 c_{1} c_{2}^{3} \geq 0$.

PROOF. We follow the same arguments used in Theorems 3.3 and 3.4 for the cases with a nondegenerate Ricci operator. From Table I, there exist eight distinct admissible degenerate Segre types for the (non-diagonal) Ricci operator of a conformally flat four-manifold of neutral signature. Among these cases, when considered for left-invariant neutral metrics of four-dimensional Lie groups, Segre types $[(1,1) 1 \overline{1}]$ and $[(1,1) 2]$ lead to a contradiction, just like we showed for most of nondegenerate Segre types in the proof of Theorem 3.3. Moreover, Segre type [(1 $\overline{1} 1 \overline{1})]$ only yields Ricci-parallel examples. In the remaining five cases, 
we can proceed as in the proof of Theorem 3.4. We found some explicit (not Ricci-parallel) examples realizing the prescribed degenerate Segre type for the Ricci operator. We can describe the general solutions for Segre types $[1,(12)]$ and $[(1,3)]$. In the remaining cases, it is really difficult to identify the underlying Lie group structure for the general solutions. For this reason, we only inserted a special solution of the general equations coming from the required form of the Ricci operator.

For the different cases (1) through (5) listed above, we now report the Ricci operator with respect to the given pseudo-orthonormal basis $\left\{e_{1}, e_{2}, e_{3}, e_{4}\right\}$, and a more explicit description of the Lie algebra:

(1): With respect to $\left\{e_{1}, e_{2}, e_{3}, e_{4}\right\}$, the Ricci operator is given by

$$
Q=\left(\begin{array}{cccc}
-4 c_{2}^{2} & 0 & 0 & 0 \\
0 & 1-4 c_{2}^{2} & -1 & 0 \\
0 & 1 & -1-4 c_{2}^{2} & 0 \\
0 & 0 & 0 & 0
\end{array}\right)
$$

So, it is easily seen that $Q$ is indeed of Segre type [1, (12)].

Moreover, time-like vector $e_{4}$ acts on the Lorentzian Lie algebra $\mathfrak{g}_{3}=\operatorname{Span}\left\{e_{1}, e_{2}, e_{3}\right\}$. With respect to the classification given in [12], this solvable Lie algebra, depending on the values of $c_{1}$ and $c_{2}$, corresponds to either the case (1) or the case (4) (with three equal eigenvalues for the self-adjoint operator $L$ and a two-dimensional eigenspace). So, it is the Lie algebra of $E(1,1)$. The same conclusion follows independently from the classification given in [1].

(2): In this case, we find

$$
Q=\left(\begin{array}{cccc}
0 & 0 & 0 & 0 \\
0 & 1 & -1 & 0 \\
0 & 1 & -1 & 0 \\
0 & 0 & 0 & 0
\end{array}\right)
$$

Hence, $Q$ is of Segre type $[(1,12)]$.

Next, the null vector $e_{2}-e_{3}$ acts on the Lie algebra $\mathfrak{g}_{3}=\operatorname{Span}\left\{e_{1}, e_{4}, u=e_{2}+e_{3}\right\}$. Moreover,

$$
\left[e_{1}, u\right]=0, \quad\left[u, e_{4}\right]=0, \quad\left[e_{1}, e_{4}\right]=0,
$$

so that $\mathfrak{g}_{3}$ is abelian, that is, $\mathfrak{g}=\mathfrak{r} \ltimes \mathfrak{r}^{3}$.

(3): The Ricci operator is given by

$$
Q=\left(\begin{array}{cccc}
1 & 0 & -1 & 0 \\
0 & 1 & 0 & -1 \\
1 & 0 & -1 & 0 \\
0 & 1 & 0 & -1
\end{array}\right)
$$

which is of Segre type [(22)]. 
Moreover, the null vector $e_{1}-e_{3}$ acts on $\mathfrak{g}_{3}=\operatorname{Span}\left\{u:=e_{1}+e_{3}, e_{2}, e_{4}\right\}$. Since

$$
\left[u, e_{2}\right]=-\left[u, e_{4}\right]=-2 c_{1} e_{2}-2 c_{1} e_{4}, \quad\left[e_{2}, e_{4}\right]=0,
$$

the derived algebra of $\mathfrak{g}_{3}$ is one-dimensional, and $\mathfrak{g}_{3}$ is the Lie algebra of the Heisenberg group.

(4): We have

$$
Q=\left(\begin{array}{cccc}
3 c_{1}^{2} & \sqrt{2} & 0 & 0 \\
\sqrt{2} & 3 c_{1}^{2} & -\sqrt{2} & 0 \\
0 & \sqrt{2} & 3 c_{1}^{2} & 0 \\
0 & 0 & 0 & 3 c_{1}^{2}
\end{array}\right) .
$$

Thus, $Q$ is of Segre type [(13)].

Next, time-like vector $e_{4}$ acts on the Lorentzian Lie algebra $\mathfrak{g}_{3}=\operatorname{Span}\left\{e_{1}, e_{2}, e_{3}\right\}$, which, by the classification of [12], is the Lie algebra of $E(1,1)$.

(5): In this case,

$$
Q=\left(\begin{array}{cccc}
-3 c_{2}^{2} & 0 & 0 & 0 \\
0 & -3 c_{2}^{2} & \sqrt{2} & 0 \\
0 & -\sqrt{2} & -3 c_{2}^{2} & \sqrt{2} \\
0 & 0 & \sqrt{2} & -3 c_{2}^{2}
\end{array}\right) .
$$

So, $Q$ is of Segre type $[(1,3)]$.

Moreover, space-like vector $e_{1}$ acts on the Lie algebra $\mathfrak{g}_{3}=\operatorname{Span}\left\{e_{1}, e_{2}, e_{3}\right\}$ (Lorentzian, but of signature $(+,-,-)$ ), which, by the classification of [12] (or [1]), is the Lie algebra of $E(1,1)$.

We can proceed in the same way for Lorentzian metrics, proving the following.

THEOREM 4.4. Let $(M, g)$ be a conformally flat, not Ricci-parallel, homogeneous Lorentzian four-manifold, with a degenerate and not diagonalizable Ricci operator $Q$. If $(M, g)$ has trivial isotropy, then it is locally isometric to a Lie group G, equipped with a left-invariant Lorentzian metric, and $Q$ is of Segre type either $[(11,2)]$ or $[(1,3)]$. More precisely, we list below an explicit description of the Lie algebra $\mathfrak{g}$ of $G$, with respect to a pseudo-orthonormal basis $\left\{e_{1}, e_{2}, e_{3}, e_{4}\right\}$, with $e_{4}$ time-like:

(1) Segre type $[(11,2)]$ : an explicit solution is given by the solvable Lie group $G=$ $\boldsymbol{R} \ltimes H$, whose Lie algebra $\mathfrak{g}$ is described by

$$
\begin{aligned}
& {\left[e_{1}, e_{2}\right]=c_{1} e_{3}+c_{1} e_{4},} \\
& {\left[e_{1}, e_{3}\right]=-\left[e_{1}, e_{4}\right]=-\left(1 / 2 c_{2}\right) e_{1}-c_{1} e_{2}-c_{3} e_{3}-c_{3} e_{4},} \\
& {\left[e_{3}, e_{4}\right]=\left(\left(2 c_{2}^{2}+1\right) / 2 c_{2}\right)\left(e_{3}+e_{4}\right),} \\
& {\left[e_{2}, e_{3}\right]=-\left[e_{2}, e_{4}\right]=-c_{2} e_{2}+c_{4} e_{3}+c_{4} e_{4},}
\end{aligned}
$$


for any real constants $c_{1}, c_{3}, c_{4}$ and $c_{2} \neq 0$. The Ricci operator is given by

$$
Q=\left(\begin{array}{cccc}
0 & 0 & 0 & 0 \\
0 & 0 & 0 & 0 \\
0 & 0 & 1 & -1 \\
0 & 0 & 1 & -1
\end{array}\right)
$$

and the light-like vector $e_{3}-e_{4}$ acts on the Lie algebra $\mathfrak{g}_{3}=\operatorname{Span}\left\{e_{1}, e_{2}, e_{3}+e_{4}\right\}$ of $H$.

(2) Segre type $[(1,3)]: G$ is the solvable Lie group $=\boldsymbol{R} \ltimes E(1,1)$, whose Lie algebra $\mathfrak{g}$ is described by

$$
\begin{aligned}
& {\left[e_{1}, e_{2}\right]=\left(c_{1}+\sqrt{2} c_{2}\right) e_{2}-\left(1 / 4 c_{2}\right) e_{3}-\left(c_{1}+\sqrt{2} c_{2}\right) e_{4},} \\
& {\left[e_{1}, e_{3}\right]=-\left(3 / 4 c_{2}\right) e_{2}+\sqrt{2} c_{2} e_{3}+\left(3 / 4 c_{2}\right) e_{4},} \\
& {\left[e_{1}, e_{4}\right]=\left(c_{1}-\sqrt{2} c_{2}\right) e_{2}-\left(1 / 4 c_{2}\right) e_{3}+\left(-c_{1}+\sqrt{2} c_{2}\right) e_{4},} \\
& {\left[e_{2}, e_{4}\right]=\left(\phi / c_{2}\right)\left(e_{2}-e_{4}\right),} \\
& {\left[e_{2}, e_{3}\right]=-\left[e_{3}, e_{4}\right]=\left(3 \sqrt{2} \phi / 16 c_{2}^{3}\right) e_{2}-\left(\phi / 2 c_{2}\right) e_{3}-\left(3 \sqrt{2} \phi / 16 c_{2}^{3}\right) e_{4},}
\end{aligned}
$$

where $\phi= \pm \sqrt{4 \sqrt{2} c_{1} c_{2}^{3}-1}$, for any real constants $c_{1}, c_{2} \neq 0$ such that $4 \sqrt{2} c_{1} c_{2}^{3}-1 \geq 0$. The Ricci operator is given by

$$
Q=\left(\begin{array}{cccc}
-6 c_{2}^{2} & 0 & 0 & 0 \\
0 & -6 c_{2}^{2} & \sqrt{2} & 0 \\
0 & \sqrt{2} & -6 c_{2}^{2} & \sqrt{2} \\
0 & 0 & -\sqrt{2} & -6 c_{2}^{2}
\end{array}\right),
$$

and $e_{1}$, space-like, acts on the Lorentzian Lie algebra $\mathfrak{g}_{3}=\operatorname{Span}\left\{e_{2}, e_{3}, e_{4}\right\}$ of $E(1,1)$.

5. Cases with degenerate Ricci operator and nontrivial isotropy. It is a wellknown fact that the same homogeneous space can admit several different realizations as a coset space. In particular, condition $\mathfrak{h} \neq 0$ does not exclude the possibility that this manifold is (also) isometric to some Lie group. A very basic example is given by the three-sphere $S^{3}$ : its Ricci operator is obviously degenerate, and $\mathfrak{h} \neq 0$ is three-dimensional, but $S^{3}$ is also isometric to the Lie group $S U(2)$.

However, condition $\mathfrak{h} \neq 0$ ensures that $M$ also corresponds to one of the examples listed in [10]. Thus, in order to complete the classification of four-dimensional conformally flat homogeneous pseudo-Riemannian manifolds, we consider the classification of homogeneous pseudo-Riemannian four-manifolds given in [10]. We start illustrating the argument used in [10] to describe the Ricci curvature of a homogeneous pseudo-Riemannian four-manifold.

Consider a homogeneous manifold $M=G / H$ (with $H$ connected), the Lie algebra $\mathfrak{g}$ of $G$ and the isotropy subalgebra $\mathfrak{h}$, and $\mathfrak{m}=\mathfrak{g} / \mathfrak{h}$ the factor space, which is identified with a subspace of $\mathfrak{g}$ complementary to $\mathfrak{h}$. The pair $(\mathfrak{g}, \mathfrak{h})$ uniquely defines the isotropy representation

$$
\psi: \mathfrak{g} \rightarrow \mathfrak{g l}(\mathfrak{m}), \quad \psi(x)(y)=[x, y]_{\mathfrak{m}} \quad \text { for all } x \in \mathfrak{g}, \quad y \in \mathfrak{m} .
$$


Given a basis $\left\{h_{1}, \ldots, h_{r}, u_{1}, \ldots, u_{n}\right\}$ of $\mathfrak{g}$, where $\left\{h_{j}\right\}$ and $\left\{u_{i}\right\}$ are bases of $\mathfrak{h}$ and $\mathfrak{m}$, respectively, a bilinear form on $\mathfrak{m}$ is determined by the matrix $g$ of its components with respect to the basis $\left\{u_{i}\right\}$, and is invariant if and only if ${ }^{t} \psi(x) \circ g+g \circ \psi(x)=0$ for all $x \in \mathfrak{g}$. Invariant pseudo-Riemannian metrics $g$ on the homogeneous space $M=G / H$ are in a one-to-one correspondence with nondegenerate invariant symmetric bilinear forms $g$ on $\mathfrak{m}$ [10].

Next, $g$ uniquely defines its invariant linear Levi-Civita connection, described in terms of the corresponding homomorphism of $\mathfrak{h}$-modules $\Lambda: \mathfrak{g} \rightarrow \mathfrak{g l}(\mathfrak{m})$ such that $\Lambda(x)\left(y_{\mathfrak{m}}\right)=$ $[x, y]_{\mathfrak{m}}$ for all $x \in \mathfrak{h}, y \in \mathfrak{g}$. Explicitly, one has

$$
\Lambda(x)\left(y_{\mathfrak{m}}\right)=\frac{1}{2}[x, y]_{\mathfrak{m}}+v(x, y) \quad \text { for all } x, y \in \mathfrak{g},
$$

where $v: \mathfrak{g} \times \mathfrak{g} \rightarrow \mathfrak{m}$ is the $\mathfrak{h}$-invariant symmetric mapping uniquely determined by

$$
2 g\left(v(x, y), z_{\mathfrak{m}}\right)=g\left(x_{\mathfrak{m}},[z, y]_{\mathfrak{m}}\right)+g\left(y_{\mathfrak{m}},[z, x]_{\mathfrak{m}}\right) \quad \text { for all } x, y, z \in \mathfrak{g} .
$$

The curvature tensor is then determined by

$$
\begin{aligned}
R: \mathfrak{m} \times \mathfrak{m} & \rightarrow \mathfrak{g l}(\mathfrak{m}) \\
(x, y) & \mapsto[\Lambda(x), \Lambda(y)]-\Lambda([x, y]) .
\end{aligned}
$$

Finally, the Ricci tensor $\varrho$ of $g$, described in terms of its components with respect to $\left\{u_{i}\right\}$, is given by

$$
\varrho\left(u_{i}, u_{j}\right)=\sum_{r=1}^{4} R_{r i}\left(u_{r}, u_{j}\right), \quad i, j=1, \ldots, 4,
$$

the Ricci operator $Q$ is uniquely determined by condition $g(Q(X), Y)=\varrho(X, Y)$, and the scalar curvature is calculated as the trace of $Q$. We then have all the needed information to check whether the equation (2.1) holds, that is, if $M^{4}=G / H$ is conformally flat.

We applied the above argument to all the spaces included in Komrakov's classification [10] of four-dimensional homogeneous pseudo-Riemannian with nontrivial isotropy, and checked the possible forms for the Ricci operator. The results we obtained are resumed in the following.

THEOREM 5.1. Let $(M, g)$ be a conformally flat homogeneous, not locally symmetric pseudo-Riemannian four-manifold with nontrivial isotropy, whose Ricci operator $Q$ is not diagonalizable and degenerate. Then, $Q$ is of Segre type either [(22)], [(1,12)], or $[(11,2)]$. Conformally flat homogeneous, not locally symmetric pseudo-Riemannian fourmanifolds with Ricci operator of these Segre types are listed in Tables III, IV and V.

PROOF. Following the notation and the classification used in [10], the space identified by the type $n . m^{k}: q$ is the one corresponding to the $q$-th pair $(\mathfrak{g}, \mathfrak{h})$ of type $n . m^{k}$, where $n=\operatorname{dim}(\mathfrak{h})(=1, \ldots, 6), m$ is the number of the complex subalgebra $\mathfrak{h}^{C}$ of $\mathfrak{s o}(4, \boldsymbol{C})$ and $k$ is the number of the real form of $\mathfrak{h}^{C}$. 
We list in these tables all conformally flat pseudo-Riemannian homogeneous fourmanifolds with nontrivial isotropy and non diagonalizable Ricci operator, which are not locally symmetric. In each of the different cases, $\left\{u_{1}, u_{2}, u_{3}, u_{4}\right\}$ is the basis of $\mathfrak{m}$ used in [10] in the description of the quotient space $M=G / H$, and $\left\{\omega_{1}, \omega_{2}, \omega_{3}, \omega_{4}\right\}$ the corresponding dual basis of one-forms. Moreover, $\omega_{i} \omega_{j}$ and $u_{i} \omega_{j}$ respectively denote the symmetric tensor product of $\omega_{i}$ and $\omega_{j}$, and the tensor product $u_{i} \otimes \omega_{j}$.

\begin{tabular}{|c|c|c|}
\hline Case & Invariant metric & Ricci operator \\
\hline $1.3^{1}: 2$ & $-2 a \omega_{1} \omega_{4}+2 a \omega_{2} \omega_{3}+b \omega_{3} \omega_{3}+2 c \omega_{3} \omega_{4}$ & $\begin{array}{l}\frac{\lambda+1}{2 a}\left(-u_{1} \omega_{3}+u_{2} \omega_{4}\right)+\frac{1}{2 a} u_{1} \omega_{4} \\
-\frac{\lambda^{2}+1}{2 a} u_{2} \omega_{3}, \quad \lambda \neq 0\end{array}$ \\
\hline $1.3^{1}: 4$ & $2 a\left(-\omega_{1} \omega_{4}+\omega_{2} \omega_{3}\right)+b \omega_{3} \omega_{3}+2 c \omega_{3} \omega_{4}$ & $\begin{array}{l}\frac{\lambda}{a}\left(-u_{1} \omega_{3}+u_{2} \omega_{4}\right)+\frac{1}{2 a} u_{1} \omega_{4} \\
+\frac{1-\lambda^{2}}{2 a} u_{2} \omega_{3}\end{array}$ \\
\hline $1.3^{1}: 5$ & $\begin{array}{l}2 a\left(-\omega_{1} \omega_{4}+\omega_{2} \omega_{3}\right)+\frac{2 c \lambda \mu-d \lambda^{2}-\mu d-2 c \lambda}{\mu(\mu-1)} \omega_{3} \omega_{3} \\
+2 c \omega_{3} \omega_{4}+d \omega_{4} \omega_{4}\end{array}$ & $\begin{array}{l}\frac{\lambda \mu}{2 a}\left(u_{1} \omega_{3}-u_{2} \omega_{4}\right)+\frac{\mu^{2}-2 \mu}{2 a} u_{1} \omega_{4} \\
-\frac{\lambda^{2} \mu+\lambda^{2}-2 \mu+4}{2 a(\mu-1)} u_{2} \omega_{3}, \quad(\lambda, \mu) \neq(0,2)\end{array}$ \\
\hline $1.3^{1}: 7$ & $\begin{array}{l}2 a\left(-\omega_{1} \omega_{4}+\omega_{2} \omega_{3}\right)+b \omega_{3} \omega_{3}+2 c \omega_{3} \omega_{4} \\
+(b \lambda-2 c) \omega_{4} \omega_{4}\end{array}$ & $\begin{array}{l}\frac{\lambda}{a(1+\lambda)}\left(-u_{1} \omega_{3}+u_{2} \omega_{4}+u_{1} \omega_{4}\right. \\
\left.+\frac{1-\lambda}{2 \lambda} u_{2} \omega_{3}\right), \quad \lambda \neq 0\end{array}$ \\
\hline $1.3^{1}: 15$ & $2 a\left(-\omega_{1} \omega_{4}+\omega_{2} \omega_{3}\right)-d \omega_{3} \omega_{3}+2 c \omega_{3} \omega_{4}+d \omega_{4} \omega_{4}$ & $\frac{1}{2 a}\left(2 u_{1} \omega_{4}+u_{2} \omega_{3}\right)$ \\
\hline $1.3^{1}: 16$ & $2 a\left(-\omega_{1} \omega_{4}+\omega_{2} \omega_{3}\right)+d \omega_{3} \omega_{3}+2 c \omega_{3} \omega_{4}+d \omega_{4} \omega_{4}$ & $\frac{1}{2 a}\left(-2 u_{1} \omega_{4}+u_{2} \omega_{3}\right)$ \\
\hline $1.3^{1}: 24$ & $\begin{array}{l}2 a\left(-\omega_{1} \omega_{4}+\omega_{2} \omega_{3}\right)+2 d\left(\lambda^{2}-\lambda\right) \omega_{3} \omega_{3}+2 c \omega_{3} \omega_{4} \\
+d \omega_{4} \omega_{4}\end{array}$ & $\begin{array}{l}\frac{\lambda-2}{2 a(\lambda-1)} u_{1} \omega_{4}-\frac{3 \lambda^{2}-8 \lambda+4}{2 a} u_{2} \omega_{3}, \\
\lambda \neq 0, \frac{2}{3}, 2\end{array}$ \\
\hline $1.3^{1}: 25$ & $\begin{array}{l}2 a\left(-\omega_{1} \omega_{4}+\omega_{2} \omega_{3}\right)-2 d\left(\lambda^{2}-\lambda\right) \omega_{3} \omega_{3}+2 c \omega_{3} \omega_{4} \\
+d \omega_{4} \omega_{4}\end{array}$ & $\begin{array}{l}\frac{2-\lambda}{2 a(\lambda-1)} u_{1} \omega_{4}-\frac{3 \lambda^{2}-8 \lambda+4}{2 a} u_{2} \omega_{3}, \\
\lambda \neq 0, \frac{2}{3}, 2\end{array}$ \\
\hline $1.3^{1}: 28$ & $\begin{array}{l}2 a\left(-\omega_{1} \omega_{4}+\omega_{2} \omega_{3}\right)+2 d \omega_{3} \omega_{3}+2 c \omega_{3} \omega_{4} \\
+d \omega_{4} \omega_{4}\end{array}$ & $\frac{1}{2 a}\left(u_{1} \omega_{4}-3 u_{2} \omega_{3}\right)$ \\
\hline $1.3^{1}: 29$ & $\begin{array}{l}2 a\left(-\omega_{1} \omega_{4}+\omega_{2} \omega_{3}\right)-2 d \omega_{3} \omega_{3}+2 c \omega_{3} \omega_{4} \\
+d \omega_{4} \omega_{4}\end{array}$ & $-\frac{1}{2 a}\left(u_{1} \omega_{4}+3 u_{2} \omega_{3}\right)$ \\
\hline $1.3^{1}: 30$ & $\begin{array}{c}2 a\left(-\omega_{1} \omega_{4}+\omega_{2} \omega_{3}\right)+b\left(\lambda^{2}-\lambda\right) \omega_{3} \omega_{3} \\
-(b \mu+d \lambda-d-b) \omega_{3} \omega_{4}+d \omega_{4} \omega_{4}\end{array}$ & $\begin{array}{l}\frac{\mu^{2} \lambda^{2}-\lambda^{2}-\lambda \mu+\lambda-\mu^{2}+\mu}{2 a(-\lambda-\mu+\lambda \mu)}\left(u_{2} \omega_{4}-u_{1} \omega_{3}\right) \\
+\frac{\lambda \mu-\mu^{3}+\mu^{3} \lambda-\lambda-\mu^{2} \lambda-\mu+2 \mu^{2}}{2 a(-\lambda-\mu+\lambda \mu)} u_{1} \omega_{4} \\
-\frac{2 \lambda^{2}-\lambda-\lambda^{3}+\lambda \mu+\lambda^{3} \mu-\mu-\lambda^{2} \mu}{2 a(-\lambda-\mu+\lambda \mu)} u_{2} \omega_{3} \\
\lambda, \mu \neq 1\end{array}$ \\
\hline
\end{tabular}

TABLE III. Non-symmetric examples with $\mathbf{Q}$ of Segre type [(22)]. 


\begin{tabular}{|c|c|c|}
\hline Case & Invariant metric & Ricci operator \\
\hline $1.1^{1}: 1$ & $2 a \omega_{1} \omega_{3}+2 c \omega_{2} \omega_{4}+d \omega_{4} \omega_{4}$ & $\frac{a^{2}-c^{2}}{2 a^{2} c} u_{2} \omega_{4}, \quad a \neq \pm c$ \\
\hline $1.1^{1}: 2$ & $2 a \omega_{1} \omega_{3}+2 c \omega_{2} \omega_{4}+d \omega_{4} \omega_{4}$ & $\frac{2(p-1)}{b} u_{2} \omega_{4}, \quad p \neq 0,1$ \\
\hline $1.3^{1}: 5$ & $2 a\left(-\omega_{1} \omega_{4}+\omega_{2} \omega_{3}\right)+b \omega_{3} \omega_{3}+2 c \omega_{3} \omega_{4}-\frac{2 c}{\lambda} \omega_{4} \omega_{4}$ & $\frac{\lambda^{2}+4}{2 a} u_{2} \omega_{3}, \quad \lambda \neq 0=\mu$ \\
\hline $1.3^{1}: 7$ & $2 a\left(-\omega_{1} \omega_{4}+\omega_{2} \omega_{3}\right)+b \omega_{3} \omega_{3}+2 c \omega_{3} \omega_{4}-2 c \omega_{4} \omega_{4}$ & $\frac{1}{2 a} u_{2} \omega_{3}, \quad \lambda=0$ \\
\hline $1.3^{1}: 12$ & $2 a\left(-\omega_{1} \omega_{4}+\omega_{2} \omega_{3}\right)+2 c \omega_{3} \omega_{4}+d \omega_{4} \omega_{4}$ & $\frac{(\lambda-\mu)^{2}-1}{2 a} u_{1} \omega_{4}, \quad \lambda \neq \mu \pm 1$ \\
\hline $1.3^{1}: 12$ & $2 a\left(-\omega_{1} \omega_{4}+\omega_{2} \omega_{3}\right)+b \omega_{3} \omega_{3}+2 c \omega_{3} \omega_{4}+d \omega_{4} \omega_{4}$ & $\frac{2 \mu(\mu-1)}{a} u_{1} \omega_{4}, \quad \lambda=1-\mu, \mu \neq 0,1$ \\
\hline $1.3^{1}: 12$ & $2 a\left(-\omega_{1} \omega_{4}+\omega_{2} \omega_{3}\right)+b \omega_{3} \omega_{3}+2 c \omega_{3} \omega_{4}+d \omega_{4} \omega_{4}$ & $\frac{(1+2 \lambda)(-3+2 \lambda)}{8 a} u_{1} \omega_{4}, \quad \mu=\frac{1}{2}, \lambda \neq \frac{1 \pm 2}{2}$ \\
\hline $1.3^{1}: 19$ & $2 a\left(-\omega_{1} \omega_{4}+\omega_{2} \omega_{3}\right)+2 c \omega_{3} \omega_{4}+d \omega_{4} \omega_{4}$ & $-\frac{1}{2 a} u_{1} \omega_{4}$ \\
\hline $1.3^{1}: 21$ & $2 a\left(-\omega_{1} \omega_{4}+\omega_{2} \omega_{3}\right)+2 c \omega_{3} \omega_{4}+d \omega_{4} \omega_{4}$ & $\frac{\lambda(\lambda-2)}{2 a} u_{1} \omega_{4}, \quad \lambda \neq 0,2$ \\
\hline $1.3^{1}: 21$ & $2 a\left(-\omega_{1} \omega_{4}+\omega_{2} \omega_{3}\right)+b \omega_{3} \omega_{3}+2 c \omega_{3} \omega_{4}+d \omega_{4} \omega_{4}$ & $-\frac{3}{8 a} u_{1} \omega_{4}, \quad \lambda=\frac{1}{2}$ \\
\hline $1.3^{1}: 24$ & $2 a\left(-\omega_{1} \omega_{4}+\omega_{2} \omega_{3}\right)+b \omega_{3} \omega_{3}+2 c \omega_{3} \omega_{4}+d \omega_{4} \omega_{4}$ & $\frac{2}{a} u_{1} \omega_{4}, \quad \lambda=\frac{2}{3}$ \\
\hline $1.3^{1}: 25$ & $2 a\left(-\omega_{1} \omega_{4}+\omega_{2} \omega_{3}\right)+b \omega_{3} \omega_{3}+2 c \omega_{3} \omega_{4}+d \omega_{4} \omega_{4}$ & $-\frac{2}{a} u_{1} \omega_{4}, \quad \lambda=\frac{2}{3}$ \\
\hline $1.3^{1}: 30$ & $2 a\left(\omega_{2} \omega_{3}-\omega_{1} \omega_{4}\right)+b \omega_{3} \omega_{3}+b(1-\mu) \omega_{3} \omega_{4}+d \omega_{4} \omega_{4}$ & $\frac{1-\mu^{2}}{2 a} u_{1} \omega_{4}, \quad \lambda=1 \neq \pm \mu$ \\
\hline $1.3^{1}: 30$ & $2 a\left(\omega_{2} \omega_{3}-\omega_{1} \omega_{4}\right)+b \omega_{3} \omega_{3}+d(1-\lambda) \omega_{3} \omega_{4}+d \omega_{4} \omega_{4}$ & $\frac{\lambda^{2}-1}{2 a} u_{2} \omega_{3}, \quad \pm \lambda \neq 1=\mu$ \\
\hline $1.4^{1}: 2$ & $\begin{array}{l}a\left(\omega_{2} \omega_{2}-2 \omega_{1} \omega_{3}\right)+b \omega_{3} \omega_{3}+2 c \omega_{3} \omega_{4}+d \omega_{4} \omega_{4} \\
a d<0, \quad b \neq 0\end{array}$ & $\begin{array}{l}-\frac{12}{d}\left(u_{1} \omega_{1}+u_{2} \omega_{2}+u_{3} \omega_{3}+u_{4} \omega_{4}\right) \\
-\frac{4 b}{a d} u_{1} \omega_{3}, \quad p=3\end{array}$ \\
\hline $1.4^{1}: 9$ & $\begin{array}{l}a\left(-2 \omega_{1} \omega_{3}+\omega_{2} \omega_{2}\right)+b \omega_{3} \omega_{3}+2 c \omega_{3} \omega_{4} \\
-\frac{a(4 r+1)}{4} \omega_{4} \omega_{4}\end{array}$ & $\frac{4 r-3}{8 a} u_{1} \omega_{3}, \quad r>-\frac{1}{4}, \neq \frac{3}{4}, p=-\frac{1}{2}$ \\
\hline $1.4^{1}: 10$ & $\begin{array}{l}a\left(-2 \omega_{1} \omega_{3}+\omega_{2} \omega_{2}\right)+b \omega_{3} \omega_{3}+2 c \omega_{3} \omega_{4}+d \omega_{4} \omega_{4} \\
a d<0\end{array}$ & $\frac{2 p(p+1)}{a} u_{1} \omega_{3}, \quad p \neq 0,-1, r=p+p^{2}$ \\
\hline $2.2^{1}: 2$ & $2 a\left(\omega_{1} \omega_{3}+\omega_{2} \omega_{4}\right)+b \omega_{2} \omega_{2}$ & $\frac{p^{2}-4}{2 a} u_{4} \omega_{2}, \quad p \neq 0, \pm 2$ \\
\hline $2.2^{1}: 3$ & $2 a\left(\omega_{1} \omega_{3}+\omega_{2} \omega_{4}\right)+b \omega_{2} \omega_{2}$ & $\frac{1}{2 a} u_{4} \omega_{2}$ \\
\hline $2.5^{1}: 4$ & $2 a\left(\omega_{1} \omega_{3}+\omega_{2} \omega_{4}\right)+b \omega_{3} \omega_{3}$ & $\frac{2 h-h^{2}+4 g}{2 a} u_{1} \omega_{3}, \quad 2 h-h^{2}+4 g \neq 0$ \\
\hline $3.3^{1}: 1$ & $2 a\left(\omega_{1} \omega_{3}+\omega_{2} \omega_{4}\right)+b \omega_{3} \omega_{3}$ & $-\frac{2 p}{a} u_{1} \omega_{3}, \quad p \neq 0$ \\
\hline
\end{tabular}

TABLE IV. Non-symmetric examples with $\mathbf{Q}$ of Segre type $[(1,12)]$. 


\begin{tabular}{|l|l|l|}
\hline Case & Invariant metric & Ricci operator \\
\hline $1.1^{2}: 1$ & $c\left(\omega_{1} \omega_{1}+\omega_{3} \omega_{3}\right)+2 b \omega_{2} \omega_{4}+d \omega_{4} \omega_{4}$ & $\frac{4 c^{2}+b^{2}}{2 b c^{2}} u_{2} \omega_{4}$ \\
\hline $1.1^{2}: 2$ & $c\left(\omega_{1} \omega_{1}+\omega_{3} \omega_{3}\right)+2 b \omega_{2} \omega_{4}+d \omega_{4} \omega_{4}$ & $\frac{2(p-1)}{b} u_{2} \omega_{4}, \quad p \neq 0,1$ \\
\hline $1.4^{1}: 2$ & $\begin{array}{l}a\left(-2 \omega_{1} \omega_{3}+\omega_{2} \omega_{2}\right)+b \omega_{3} \omega_{3}+2 c \omega_{3} \omega_{4} \\
+d \omega_{4} \omega_{4}, \quad a d>0, \quad b \neq 0\end{array}$ & $\begin{array}{l}-\frac{12}{d}\left(u_{1} \omega_{1}+u_{2} \omega_{2}+u_{3} \omega_{3}+u_{4} \omega_{4}\right) \\
-\frac{4 b}{d a} u_{1} \omega_{3}, \quad p=3\end{array}$ \\
\hline $1.4^{1}: 9$ & $\begin{array}{l}a\left(-2 \omega_{1} \omega_{3}+\omega_{2} \omega_{2}\right)+b \omega_{3} \omega_{3}+2 c \omega_{3} \omega_{4} \\
-\frac{a(4 r+1)}{4} \omega_{4} \omega_{4}\end{array}$ & $\frac{4 r-3}{8 a} u_{1} \omega_{3}, \quad p=-\frac{1}{2}, r<-\frac{1}{4}$ \\
\hline $1.4^{1}: 10$ & $\begin{array}{l}a\left(-2 \omega_{1} \omega_{3}+\omega_{2} \omega_{2}\right)+b \omega_{3} \omega_{3}+2 c \omega_{3} \omega_{4} \\
+d \omega_{4} \omega_{4}, \quad a d>0\end{array}$ & $\frac{2 p(p+1)}{a} u_{1} \omega_{3}, \quad p \neq 0,-1, r=p(p+1)$ \\
\hline $2.5^{2}: 2$ & $2 a \omega_{1} \omega_{3}+a\left(\omega_{2} \omega_{2}+\omega_{4} \omega_{4}\right)+b \omega_{3} \omega_{3}$ & $\frac{2\left(p+r^{2}\right)}{a} u_{1} \omega_{3}, \quad p \neq-r^{2}, s=0$ \\
\hline $3.3^{2}: 1$ & $2 a \omega_{1} \omega_{3}+a\left(\omega_{2} \omega_{2}+\omega_{4} \omega_{4}\right)+b \omega_{3} \omega_{3}$ & $\frac{2 p}{a} u_{1} \omega_{3}, \quad p \neq 0$ \\
\hline
\end{tabular}

TABLE V. Non-symmetric examples with $\mathbf{Q}$ of Segre type $[(11,2)]$

\section{REFERENCES}

[1] A. Bowers, Classification of three-dimensional real Lie algebras, available online at the webpage math.ucsd.edu/ abowers/downloads/survey/3d_Lie_alg_classify.pdf

[2] M. Brozos-Vazquez, E. Garcia-Rio, P. Gilkey, S. Nikcevic and R. Vazquez-Lorenzo, The geometry of Walker manifolds, Synth. Lect. Math. Stat 5, Morgan \& Claypool Publishers, Williston, VT, 2009.

[3] G. Calvaruso, Einstein-like metrics on three-dimensional homogeneous Lorentzian manifolds, Geom. Dedicata 127 (2007), 99-119.

[4] G. Calvaruso And A. Zaeim, Four-dimensional homogeneous Lorentzian manifolds, Monaths. Math., to appear. DOI: 10.1007/s00605-013-588-9.

[5] M. ChAichi, E. GARCiA-Rio AND Y. MATsushita, Curvature properties of four-dimensional Walker metrics, Classical Quantum Gravity 22 (2005), 559-577.

[6] M. ChAichi AND A. ZAEIM, Locally homogeneous four-dimensional manifolds of signature $(2,2)$, Math. Phys. Anal. Geom. 16 (2013), 345-361.

[7] K. HondA, Conformally flat semi-Riemannian manifolds with commuting curvature and Ricci operators, Tokyo J. Math. 26 (2003), 241-260.

[8] K. Honda AND K. TsuKADA, Three-dimensional conformally flat homogeneous Lorentzian manifolds, J. Phys. A 40 (2007), 831-851.

[9] K. Honda And K. Tsukada, Conformally flat homogeneous Lorentzian manifolds, Proceedings of the conference "GELOGRA", Granada (Spain) 2011, to appear.

[10] B. KomraKov JnR., Einstein-Maxwell equation on four-dimensional homogeneous spaces, Lobachevskii J. Math. 8 (2001), 33-165.

[11] P. R. LAW, Algebraic classification of the Ricci curvature tensor and spinor for neutral signature in four dimensions, arXiv:1008.0444v1, 2010.

[12] S. Rahmani, Métriques de Lorentz sur les groupes de Lie unimodulaires de dimension trois, J. Geom. Phys. 9 (1992), 295-302.

[13] P. RYAN, A note on conformally flat spaces with constant scalar curvature, Proceedings of the Thirteenth Biennial Seminar of the Canadian Mathematical Congress, Vol. 2 (On Differential Topology, Differential Geometry and Applications, Dalhousie Univ., Halifax, N.S., 1971), pp. 115-124, Canad. Math. Congr., 
Montreal, Que., 1972.

[14] I. M. Singer, Infinitesimally homogeneous spaces, Comm. Pure Appl. Math. 13 (1960), 685-697.

[15] H. Stephani, D. Kramer, M. Maccallum, C. Hoenselaers and E. Herlt, Exact solutions of Einstein's field equations, Cambridge Monographs on Mathematical Physics, 2nd. rev. Ed., Cambridge University Press, 2009.

[16] S. Sternberg, Lectures on differential geometry, Prentice-Hall, Englewood Cliffs, N.J., 1964.

[17] H. TAKAGI, Conformally flat Riemannian manifolds admitting a transitive group of isometries, I,II, Tohôku Math. J. 27 (1975), 103-110 and 445-451.

Dipartimento di MATEMATICA E Fisica

"E. DE GIORGI"

UNIVERSITÀ DEL SALENTO

PROV. LECCE-ARNESANO

73100 LECCE

ITALY

E-mail address: giovanni.calvaruso@unisalento.it
DePARTMENT OF MATHEMATICS

PAYAME NOOR UNIVERSITY

P.O. BOX 19395-3697

TEHRAN

IRAN

E-mail address: Zaeim@phd.pnu.ac.ir 